\title{
An improved genome assembly uncovers prolific tandem repeats in Atlantic cod
}

\author{
Ole K. Tørresen ${ }^{1 *}$, Bastiaan Star ${ }^{1}$, Sissel Jentoft ${ }^{1,2}$, William B. Reinar ${ }^{1}$, Harald Grove ${ }^{3}$, Jason R. Miller ${ }^{4}$, \\ Brian P. Walenz ${ }^{5}$, James Knight ${ }^{6}$, Jenny M. Ekholm7, Paul Peluso ${ }^{7}$, Rolf B. Edvardsen ${ }^{8}$, \\ Ave Tooming-Klunderud ${ }^{1}$, Morten Skage ${ }^{1}$, Sigbjørn Lien ${ }^{3}$, Kjetill S. Jakobsen ${ }^{1}$ \\ and Alexander J. Nederbragt ${ }^{1,9^{*}}$ (D)
}

\begin{abstract}
Background: The first Atlantic cod (Gadus morhua) genome assembly published in 2011 was one of the early genome assemblies exclusively based on high-throughput 454 pyrosequencing. Since then, rapid advances in sequencing technologies have led to a multitude of assemblies generated for complex genomes, although many of these are of a fragmented nature with a significant fraction of bases in gaps. The development of long-read sequencing and improved software now enable the generation of more contiguous genome assemblies.

Results: By combining data from Illumina, 454 and the longer PacBio sequencing technologies, as well as integrating the results of multiple assembly programs, we have created a substantially improved version of the Atlantic cod genome assembly. The sequence contiguity of this assembly is increased fifty-fold and the proportion of gap-bases has been reduced fifteen-fold. Compared to other vertebrates, the assembly contains an unusual high density of tandem repeats (TRs). Indeed, retrospective analyses reveal that gaps in the first genome assembly were largely associated with these TRs. We show that $21 \%$ of the TRs across the assembly, $19 \%$ in the promoter regions and $12 \%$ in the coding sequences are heterozygous in the sequenced individual.

Conclusions: The inclusion of PacBio reads combined with the use of multiple assembly programs drastically improved the Atlantic cod genome assembly by successfully resolving long TRs. The high frequency of heterozygous TRs within or in the vicinity of genes in the genome indicate a considerable standing genomic variation in Atlantic cod populations, which is likely of evolutionary importance.
\end{abstract}

Keywords: Assembly algorithms, Assembly consolidation, Dinucleotide repeats, Gadus morhua, Heterozygosity, Indel polymorphism, Long-read sequencing technology, Microsatellites, PacBio, Repetitive DNA

\section{Background}

The speed and affordability of sequencing and improved software, including more efficient genome assemblers, have led to a democratization of genomics, enabling individual research groups to create de novo genome assemblies [1]. The first published de novo assemblies for non-model organisms using pure massively parallel sequencing approaches (Illumina and 454) appeared in

*Correspondence: o.k.torresen@ibv.uio.no; lex.nederbragt@ibv.uio.no ${ }^{1}$ Centre for Ecological and Evolutionary Synthesis, Department of Biosciences, University of Oslo, NO-0316 Oslo, Norway

${ }^{9}$ Biomedical Informatics Research Group, Department of Informatics, University of Oslo, NO-0316 Oslo, Norway

Full list of author information is available at the end of the article
2010-2011 and included diverse species such as giant panda [2], turkey [3], woodland strawberry [4] and Atlantic cod [5]. Numerous genome assemblies from a myriad of non-model plants, invertebrates and vertebrates are now available, including examples of genomes that are difficult to assemble, e.g. the extremely large genomes of bread wheat [6] and Norway spruce [7], the highly heterozygous genome of oyster [8] and the tetraploid and repetitive salmon genome [9]. These genome assemblies have provided exciting new biological findings, including the first example of a vertebrate immune system, that of Atlantic cod, which lacks MHC (major histocompatibility complex) class II [5], untangling of the events of multiple hybridizations shaping the ancestral genomes of bread 
wheat prior to domestication [10] and multiple genomes resolving the avian phylogeny, their radiations and investigation of the genetic basis of complex traits [11, 12]. Despite the tremendous impact of the high throughput sequencing generated genomes, many of these assemblies are of varying completeness, depending on the purpose for which they have been obtained $[13,14]$. In the examples given above, the sizes of the scaffold sequences are usually far shorter than chromosome arm lengths. Most of these genomes have scaffold N50 lengths (i.e., half the assembly is in scaffolds of this length or longer) in the range of $400 \mathrm{kbp}-1.5 \mathrm{Mbp}$, although some avian genomes have N50 scaffold lengths up to $10 \mathrm{Mbp}$, approaching chromosome arm lengths. However, contig N50 lengths are far shorter and in the range of 3-55 kbp.

The presence of repetitive DNA is the most important factor contributing to fragmented genome assemblies $[14,15]$. Assembly algorithms might not resolve repetitive regions if they are longer than the read length, and this in particular affects the assembly of sequencing data from short-read technologies such as the Illumina platform [14-16]. Repetitive regions can be divided into two classes, interspersed and tandem repeats. Interspersed repeats, including transposable elements (TEs), occur across the genome and are present in all vertebrate genomes, comprising from 5 to $55 \%$ of their assemblies [17]. Tandem repeats (TRs) are sequences with a repeat unit repeated more than two times in tandem. Eukaryotic genomes typically consist of 0.5 to $3 \%$ TRs, and TRs can be classified into microsatellites, also called simple repeats, or short tandem repeats (STRs, 1-9 bp repeat unit size); minisatellites (10-100 bp) and satellite repeats (>100 bp repeat unit size) [18]. TRs mutate by adding or removing full repeat units and their mutation rates can be 10 to 10,000 fold higher than for the remainder of the genome [19]. The heterozygosity caused by TR mutations, in addition to other types of heterozygosity, are also likely to have confounding effects on the contiguity of genome assemblies $[14,15]$.

Long-read sequencing technologies such as PacBio and Oxford Nanopore address the drawbacks of short-read technologies by enabling read-through of larger repeat regions, and are therefore particularly well-suited for de novo genome assembly $[14,20]$. Combining moderate amounts of PacBio coverage (5-20x) with other sequencing data can dramatically improve the contiguity of an assembly [21, 22]. More extensive coverage in long reads $(>50 \mathrm{x})$ has enabled assemblies of vertebrate genomes to approach complete chromosome arms [23-25], although the associated costs are substantial. A sequencing strategy including long-reads is recommended to aid in reducing the fragmentation typical of de novo genome assemblies based on a short-read technology only. Regardless of sequencing strategy, use of a genetic linkage map, or an optical map, can place contigs or scaffolds into chromosome-sized reconstructions, called linkage groups, a prerequisite for large-scale genome comparisons between species [26].

The first release of the Atlantic cod (Gadus morhua) genome was sequenced and assembled solely with the 454 sequencing technology [5] and annotated by the Ensembl Project [27] (gadMor1). The $832 \mathrm{Mbp}$ assembly was fragmented, with a contig N50 of $2.3 \mathrm{kbp}$ and $27 \%$ of bases in gaps. The genome assembly contained $17.8 \%$ TEs and 5.9\% TRs (Supplementary Table 6 in [5]). An increased abundance of short unit size TRs at the contig termini $(32 \%)$, and at the gaps in scaffolds (24\%, Supplementary Note 7 in [5]) indicate that these repeats contributed to the observed level of fragmentation.

A more contiguous reference genome for Atlantic cod, preferably with chromosome-level reconstructions, will facilitate re-sequencing efforts addressing population genomics investigations, including the detection of structural variants, introgression and hybridization between species, as well as improve comparative genomic investigations relying on synteny. Moreover, it will also enable an annotation with more complete gene models and allow for a better understanding of the lack of sequence contiguity in gadMor1. To achieve this, we created several assemblies using different combinations of Illumina, 454 and PacBio sequencing technologies, Sanger BAC-end sequences, and a suite of assembly programs. As often is the case [28-30], no single assembly outperformed the others in all criteria (N50 contig/scaffold length, gene content, agreement with a genetic linkage map, accordance with read data), thus a reconciled assembly was created to integrate the best characteristics of four draft assemblies. This new assembly (gadMor2) has a fifty-fold improvement of the contig N50 length of gadMor1, and eight times longer scaffold N50 and one sixteenth the number of bases in gaps than gadMor1. A linkage map (personal communication, Sigbjørn Lien) was used to order and orient the scaffolds into linkage groups. The new genome assembly and annotation reveal a high content of TRs compared to other vertebrates and most notably in promoter regions and amino acid coding sequences. Many of these TRs are heterozygous, and we propose this has implications for understanding local adaptation at a population level.

\section{Results}

\section{An improved genome assembly for Atlantic cod}

In addition to already existing sequencing data for the wild-caught individual from the North East-Arctic population described in [5] ( $\sim 40 \mathrm{x}$ Roche $/ 454$ and $\sim 0.1 \mathrm{x}$ Sanger BAC-ends), we added sequencing data from Illumina ( $\sim 480$ x coverage) and PacBio ( $\sim 19 x$ coverage) (Additional file 1: Table S1) obtained from DNA isolated from the same individual. Different assembly strategies were used: 
a Newbler assembly with 454 and Sanger BAC-end sequences as input (NEWB454), an ALLPATHS-LG [31] assembly with the Illumina sequences only (ALPILM), a Celera Assembler [32] assembly with 454 and Illumina sequences (CA454ILM) and a Celera Assembler assembly with 454 paired reads, Illumina reads and raw, uncorrected PacBio reads (CA454PB) (Additional file 1: Table S1). For each of the individual assemblies, different combinations of the assembly improvement programs Pilon [33] and PBJelly [34] were applied to improve the consensus sequence and to close gaps (Additional file 1: Table S2). The properties of these assemblies were assessed using multiple tools: 1) Methods based on the mapping of read datasets to an assembly, FRC bam [35] and REAPR [36]; 2) by comparing a transcriptome to an assembly, Isoblat (using the Newbler transcriptome, see Methods) [37]; 3) by comparing the assembly to a linkage map (see Methods); 4) and determining presence and completeness of conserved eukaryotic and Actinopterygii (ray-finned fishes) gene sets, CEGMA [38] and BUSCO [39] (Additional file 1: Table S2).

Based on these evaluations, each assembly had distinct properties, and none is superior for all metrics. For instance, the NEWB454 assembly has the longest scaffold N50 and the lowest number of conflict sequences (Fig. 1, Table 1). In contrast, the CA454PB outperforms the other assemblies based on contig N50, yet has a lower scaffold N50 and higher number of sequences conflicting with the linkage map (sequences that map to two linkage groups) (Table 1). Existing assembly reconciliation tools are limited to combining two assemblies $[40,41]$ and do not perform satisfactorily. To obtain the best possible assembly, i.e., to integrate the information recovered by the different

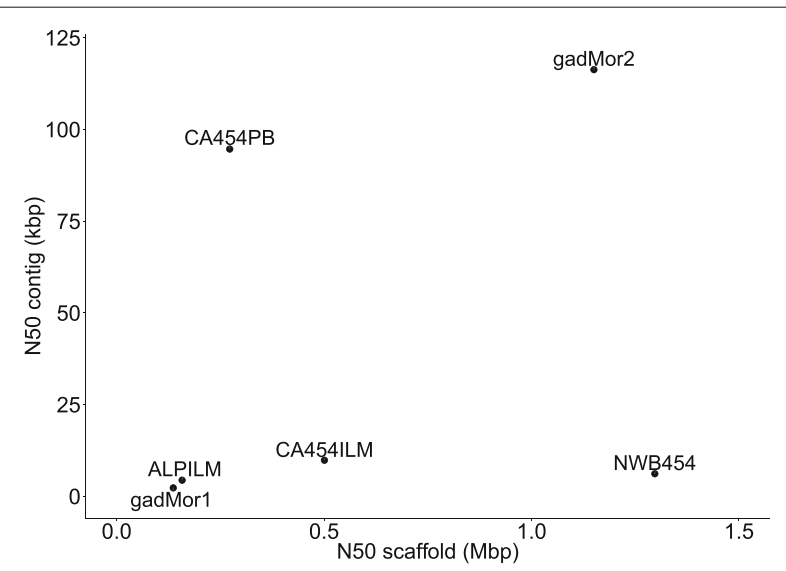

Fig. 1 Contig and scaffold N50 lengths of the different cod assemblies. gadMor2 was created by following the sequences in CA454ILM in a path through a graph created from a multiple alignment of the four original assemblies, and outputting the contig sequences from CA454PB for each alignment. NEWB454 and ALPILM were used to extend the scaffolds, see Table 1 assemblies, we developed a novel assembly reconciliation method. This method involved an all-against-all alignment of the assemblies using Mugsy [42] after splitting the different assemblies in locations where they were in conflict with the linkage map (see Methods) and removing sequences shorter than $1000 \mathrm{bp}$. The resulting alignment graph structure was traversed following the path from one of the original assemblies (CA454ILM, the one with the most genes found with CEGMA and BUSCO), yielding the sequence from the assembly with the least gaps (CA454PB), while using the alignments with ALPILM and NEWB454 in the graph to close gaps and extend scaffolds. The scaffold module from SGA [43] was applied on the resulting merged assembly using all paired reads (Illumina, 454 and sequenced BAC-ends), and Pilon [33] was used to improve per-base accuracy and to close or reduce gaps. The resulting assembly was ordered and oriented based on a linkage map of 9355 SNPs (personal communication, Sigbjørn Lien) placing 93\% of the sequences into 23 linkage groups (Additional file 1: Table S3). Comparisons of assembly statistics for the final, reconciled assembly (gadMor2) and the original four (CA454ILM, CA454PB, ALPILM and NEWB454), show that gadMor2 outperforms all other assemblies on all quality metrics apart from scaffold N50 (ranked $2^{\text {nd }}$ ) and CEGMA gene content (ranked $3^{\text {rd }}$, Table 1, Fig. 1). Based on an overall assessment of quality, gadMor2 combines the best features of each of the four original assemblies without loss of quality (Table 1).

The gadMor2 assembly has a fifty-fold longer contig N50 and eight-fold longer scaffold N50 compared to the gadMor1 assembly [5]. This has dramatic consequences for the sequence contiguity; for instance, a 100kbp region containing the HoxC cluster is a single contig in gadMor2, while it previously consisted of 21 contigs and 20 gaps in gadMor1 (Fig. 2).

\section{Genome size}

Estimation of genome size with odd-sized k-mers from 17 to 31 with SGA PreQC [44] on the 300 bp insert size, 100 bp length, paired end Illumina reads (about 150x coverage), resulted in a genome estimate of $613 \mathrm{Mbp} \pm 11 \mathrm{Mbp}$ (Additional file 1: Table S4). The assembler ALLPATHSLG estimated the genome to be $651 \mathrm{Mbp}$ based on the k-mer distribution of the $180 \mathrm{bp}$ insert size, $100 \mathrm{bp}$ length, paired end Illumina reads (about 52x coverage). Both estimates are lower than previous ones based on Feulgen Image Analysis Densitometry at $0.93 \mathrm{pg}$ or $910 \mathrm{Mbp}$ $[45,46]$ and a k-mer analysis based on 454 reads, which resulted in a $830 \mathrm{Mbp}$ estimate [5]. Although the assembly size of the gadMor1 at Ensembl is $832 \mathrm{Mbp}$ with $26.9 \%$ gaps [5], the amount of sequence in contigs is 608 Mbp (224 Mbp in gaps), considerably closer to the SGA PreQC estimate. The likely explanation for the large size 
Table 1 Overview of assembly statistics

\begin{tabular}{|c|c|c|c|c|c|c|c|c|c|}
\hline Assembly & $\begin{array}{l}\text { Total size } \\
\text { assembly } \\
\text { (Mbp) }\end{array}$ & $\begin{array}{l}\text { N50 } \\
\text { contig } \\
\text { (kbp) }\end{array}$ & $\begin{array}{l}\text { N50 } \\
\text { scaffold } \\
\text { (Mbp) }\end{array}$ & $\begin{array}{l}\text { Percentage } \\
\text { gap bases }\end{array}$ & CEGMA $^{a}$ & BUSCO $^{b}$ & REAPRC & FRC bamd & $\begin{array}{l}\text { Potential } \\
\text { conflict } \\
\text { (sequences) }^{\mathrm{e}}\end{array}$ \\
\hline $\operatorname{gadMor1}^{f}$ & 832 & 2.3 & 0.14 & 26.9 & 444 (96.9\%) & 3308 (89.4\%) & 2547 & 4210772 & 76 \\
\hline ALPILM & 660 & 4.4 & 0.16 & 28.7 & $424(92.6 \%)$ & 3016 (81.6\%) & 19787 & 2182096 & 122 \\
\hline NEWB454 & 656 & 6.2 & 1.30 & 24.4 & 435 (95.0\%) & 3109 (84.1\%) & 18117 & 2044008 & 26 \\
\hline CA454ILM & 647 & 9.9 & 0.50 & 3.49 & 447 (97.5\%) & 3379 (91.4\%) & 7406 & 1351500 & 96 \\
\hline CA454PB & 682 & 95 & 0.27 & 1.62 & 431 (94.1\%) & 3310 (89.5\%) & 8617 & 1508054 & 188 \\
\hline gadMor $2^{g}$ & 643 & 116 & 1.15 & 1.69 & 435 (95.0\%) & 3447 (93.2\%) & 7359 & 1248792 & 15 \\
\hline
\end{tabular}

${ }^{\mathrm{a} C E G M A}$ annotates 458 highly conserved eukaryotic genes

${ }^{b}$ BUSCO annotates 3,698 actinopterygii specific genes

${ }^{C}$ REAPR analyses the discordance between the expected order, orientation and distance of mapped paired reads, with detected potential errors, fewer is better

${ }^{\mathrm{d}} F R C^{\text {bam }}$ uses a similar approach as REAPR, with total number of features (i.e., potential assembly problems), fewer is better

eNumber of sequences mapping to more than one linkage group or to multiple linkage groups, fewer is better

fFrom [5]

993\% of the gadMor2 assembly is additionally oriented and ordered into 23 linkage groups (Additional file 1: Table S3)

of gadMor1 is that many of the contigs could not be placed into a scaffold, and a gap was created at that locus instead. These unplaced contigs are included in the output, resulting in loci represented twice in the assembly, once as a gap and once as a contig. The assemblies created in this study all span approximately $650 \mathrm{Mbp}$, which is similar to the ALLPATHS-LG estimation. $650 \mathrm{Mbp}$ is $71 \%$ of the earlier estimation based on Feulgen Image Analysis Densitometry, and in line with similar results in platyfish (70-89\% of earlier estimations) [47] and in northern pike (64-100\%) [48]. In addition, CEGMA [38] and BUSCO [39] find $89-93 \%$ of conserved genes (Table 1), likely reflecting the approximate completeness of the genome assembly.

\section{Annotation}

We annotated 83,505 gene models with MAKER2 $[49,50]$, obtaining a final set of 23,243 predicted genes after discarding gene models with low support (see Methods). Compared to gadMor1 (20,095 predictions) [5], the gadMor2 annotation contains more predicted genes and significantly more sequence in the predicted transcriptome (32.2 Mbp and 52.9 Mbp, respectively). The predicted transcripts are substantially longer and without gaps (Table 2). A genome browser enabling access to the genome and the annotation is available [51].

\section{Heterozygosity}

Illumina paired-end reads with $300 \mathrm{bp}$ insert size and $100 \mathrm{bp}$ read length were mapped to the gadMor2 assembly using BWA-MEM [52], and 2,621,997 SNPs (single nucleotide polymorphisms), 90,292 MNPs (multiple nucleotide polymorphisms), 631,063 indels (insertions and deletions) and 169,181 complex regions (composite insertion and substitution events) with quality $\geq 20$ were called using FreeBayes [53]. With 2,621,997 SNPs, this corresponds to a (SNP) heterozygosity rate of $4.07 \times 10^{-3}$ (one segregating site every $246 \mathrm{bp}$ ). The indel rate in Atlantic cod is $0.98 \times 10^{-3}$ (one indel every $1020 \mathrm{bp}$ on average, Table 3).

We also called indels based on PacBio sequencing reads using blasr [54] and PBHoney [55]. 70,278 indels of size $\geq 20 \mathrm{bp}$ were found, at a rate of $0.1 \times 10^{-3}$ indels/base, or one indel $\geq 20$ bp every 10,000 bp on average.

\section{Repeat content}

We created a repeat library using a combination of RepeatModeler [56], LTRharvest [57], LTRdigest [58] and TransposonPSI [59] and known eukaryotic TE sequences from RepBase [60] (see Methods). This library masked $31.3 \%$ of the genome assembly (Table 4 ), with $22.9 \%$ classified as interspersed repeats (most often TEs) and $8.0 \%$ as TRs (ranging from dinucletide to hexanucleotide repeats,

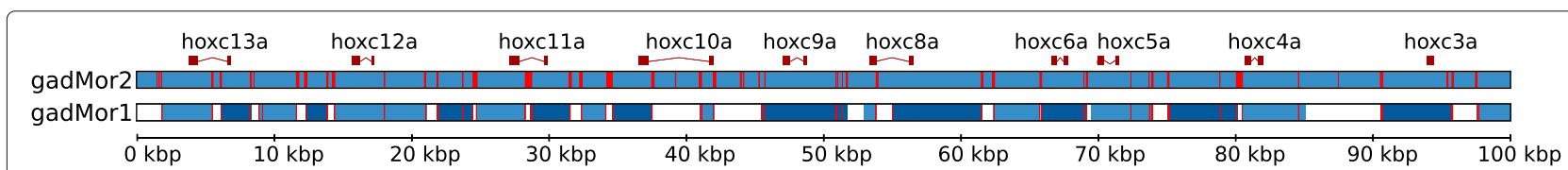

Fig. 2 The HoxC cluster in gadMor1 and gadMor2. Blocks of dark and light blue are contig sequences, white blocks are gaps and red lines are tandem repeats. Gene models are sketched at the top of the figure. This region is a single contig in gadMor2 and 21 contigs in gadMor1. Tandem repeats are at the borders between almost all gaps and contigs in gadMor1 
Table 2 Comparison between the gene annotations of gadMor1 and gadMor2

\begin{tabular}{llllll}
\hline Assembly & $\begin{array}{l}\text { Total size } \\
\text { transcriptome }(\mathrm{Mbp})^{\mathrm{a}}\end{array}$ & Number of genes & N50 length (bp) ${ }^{\mathrm{b}}$ & $\begin{array}{l}\text { Amount gap bases }_{(\mathrm{Mbp})^{\mathrm{c}}} \\
\text { gadMor1 }\end{array} \mathbf{B U S C O}^{\mathrm{d}}$ \\
gadMor2 & $32.2(24.8)$ & $22618^{\mathrm{e}}$ & $1854(1398)$ & 1.7 & $2947(79.7 \%)$ \\
\hline
\end{tabular}

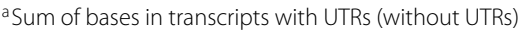

${ }^{b}$ Half the transcriptome is in sequences of this length or longer, with UTRs (without UTRs)

'Gaps represented as 'N's in annotated transcripts

${ }^{\mathrm{d}}$ Number (percentage) of conserved actinopterygii genes detected out of a total of 3,698

eWhen excluding pseudogenes, alternative transcripts, etc., the number of protein-coding genes is 20,095

fProtein-coding genes only

at least $20 \mathrm{bp}$ long), both classifications higher than for gadMor1 (17.8\% and 5.9\% respectively, Supplementary Table 6 in [5]), indicating a more complete genome assembly.

\section{TR content}

We investigated to what extent different assemblers and sequencing technologies affected the numbers of annotated TRs. Phobos [18] was used to find all TRs with a unit size of 1-50 bp, at least $13 \mathrm{bp}$ long (different from the TRs classified above), in the different cod assemblies (Fig. 3 and Table 5). Our results shows that assemblies created with the Celera Assembler have the largest amount of TRs (Fig. 3).

The most prominent class of TRs in gadMor2 is dinucleotide TRs, which make up $48.7 \%$ of all annotated repeats, followed by mononucleotide, trinucleotide and tetranucleotide repeats that comprise only $7.6 \%, 6.3 \%$ and $6.3 \%$, respectively (Fig. 4). The average length of dinucleotide repeats is $84.4 \pm 87.2 \mathrm{bp}$, at an average $97.3 \%$ identity. In total, dinucleotide repeats make up $5.7 \%$ of the entire gadMor2 assembly. NEWB454 and ALPILM have a significantly lower amount of, and shorter, TRs annotated than the two assemblies created with Celera Assembler, CA454ILM and CA454PB (Table 5).
An analysis of gadMor2 compared to all genomes in Ensembl (release 81, excluding gadMor1), including the genome of California sea hare (which contains a large amount of TRs [61]), shows that the Atlantic cod genome assembly has an approximately three-fold higher density of TRs than the genome assemblies of other vertebrates (Fig. 5, see also Additional file 1: Figure S1).

\section{TRs cause fragmentation of non-PacBio based assemblies}

To investigate the possible genomic features associated with gaps in APLILM, CA454ILM, CA454PB, NEWB454, gadMor1 and gadMor2 assemblies, we mapped the contigs from each assembly to gadMor2 and categorized the intersections between the contig termini (i.e. the positions of the terminal nucleotides of each contig) and different annotated features such as SNPs, indels, TRs, TEs and lack of sequence coverage.

For gadMor2, contig termini overlap most prominently with regions lacking read coverage by any sequencing technology, and annotated TEs. The CA454PB shows the same pattern, albeit with a larger fraction of contig termini not overlapping any annotation, suggesting that these contigs end in large repeats not resolved by any assembly. For the other assemblies, the largest fraction of contig termini overlap with TRs at percentages that are significantly

Table 3 Comparison of the SNP and indel rates of selected organisms

\begin{tabular}{|c|c|c|c|c|}
\hline Species & $\begin{array}{l}\text { SNP rate } \\
\text { (SNPs/base) }\end{array}$ & $\begin{array}{l}\text { Indel rate } \\
\text { (indels/base) }\end{array}$ & $\begin{array}{l}\text { N50 contig } \\
\text { (kbp) }\end{array}$ & $\begin{array}{l}\text { N50 scaffold } \\
\text { (Mbp) }\end{array}$ \\
\hline Atlantic cod (gadMor2) & $4.07 \times 10^{-3}$ & $0.98 \times 10^{-3}$ & 116 & 1.15 \\
\hline Stickleback ${ }^{a}$ & $1.43 \times 10^{-3}$ & NA & 83.2 & 10.8 \\
\hline Miiuy croaker ${ }^{b}$ & $2.24 \times 10^{-3}$ & $0.61 \times 10^{-3}$ & 73.3 & 1.15 \\
\hline Atlantic herring ${ }^{c}$ & $3.2 \times 10^{-3}$ & NA & 21.3 & 1.84 \\
\hline Ciona savignyid $^{\text {d }}$ & $46 \times 10^{-3}$ & NA & 12 & 0.192 \\
\hline Ciona savignyie & $46 \times 10^{-3}$ & NA & 47 & 0.989 \\
\hline
\end{tabular}

${ }^{\mathrm{a} F r o m}[68]$

bFrom [67]

cFrom [69]

dFrom [66]

eFrom [66], with haplotype assembly and merging 
Table 4 The repeat content of of the Atlantic cod genome assembly

\begin{tabular}{llll}
\hline Repeat & $\begin{array}{l}\text { Number } \\
\text { of elements }\end{array}$ & Coverage (Mbp) & Coverage $^{\mathrm{a}}(\%)$ \\
\hline LINEs & 64344 & 18.4 & 2.86 \\
LTR elements & 81087 & 22.3 & 3.47 \\
DNA elements & 269835 & 46.5 & 7.23 \\
Unclassified & 215676 & 59.2 & 9.21 \\
Total interspersed repeats & 636132 & 147.1 & 22.86 \\
Tandem repeats & 582198 & 51.2 & 7.96
\end{tabular}

${ }^{a}$ Groups of elements covering less than $1 \%$ of the genome assembly are not shown ${ }^{b}$ This is the sum of all annotated interspersed repeats, including the first four rows plus SINES

higher $(>40 \%)$ than the fraction of the gadMor2 assembly annotated as such repeats $(10.9 \%$, Table 5$)$. As TEs might be longer than the read lengths, they may represent a general challenge for most complex genomes (Fig. 6, Additional file 1: Figure S2).

\section{Heterozygous TRs}

We used lobSTR [62] to investigate the occurrence of heterozygous TRs (i.e., different repeat length between the same locus on the homologous chromosomes) in the sequenced cod genome. lobSTR is designed to analyze TRs with unit length of 1-6 bp (i.e., STRs), and uses Tandem Repeats Finder (TRF) [63] to detect them in the genome assembly. lobSTR both annotates the STRs and discovers variation in STR length. In the sequenced individual, lobSTR annotated 980,400 STRs that passed filtering $(1,182,796$ in total, see Methods), of which 47,718 were heterozygous.

Compared to Phobos (which annotated 640,938 TRs of units 1-6 bp), lobSTR annotated almost twice as

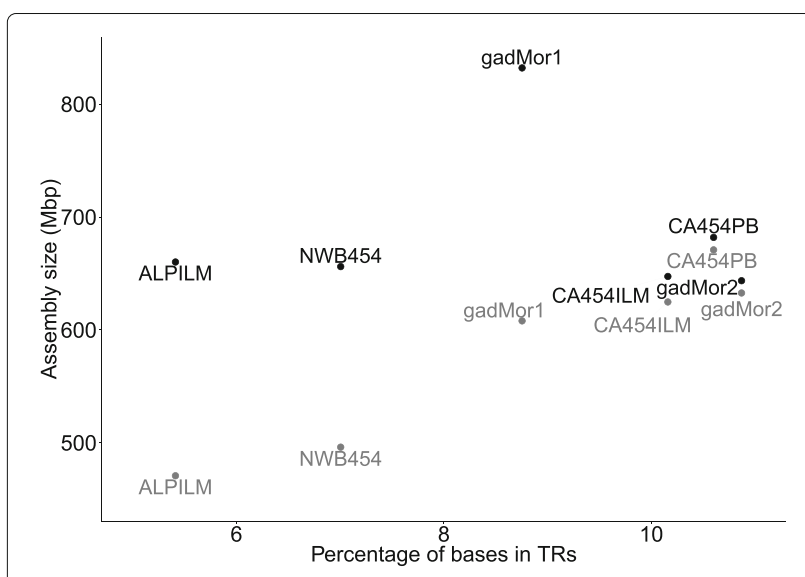

Fig. 3 The density of TRs and the size of the assembly for different cod assemblies. The different assemblies (black) are all similar in size, around $650 \mathrm{Mbp}$, with the exception of the much larger gadMor1, while the amount of sequence in contigs in the different assemblies (grey) differs substantially. The vertical distance between pairs of points for each assembly equals the amount of sequence in gaps many STRs, and the distributions of the lengths of STRs between the two programs differ largely (Additional file 1: Figure S3), with lobSTR identifying relatively short STRs, and Phobos annotating relatively long STRs. Given that lobSTR is based on the alignment of the $100 \mathrm{bp}$ Illumina reads, lobSTR's ability to detect heterozygous TRs is limited to repeats around $45 \mathrm{bp}$ in size [64], however, the average length of a TR in cod is 84.32 bp (Table 5). As an alternative to using lobSTR for detecting heterozygous TRs, we used the intersection between TRs annotated by Phobos and indels annotated by either FreeBayes (using Illumina reads, 169,635 intersections) or PBHoney (using mapped PacBio reads, 43,521 intersections). Altogether, 145,435 indels were detected in the 640,938 STRs (1-6 bp unit size) as annotated by Phobos, about three times as many as annotated by lobSTR. For TRs of unit sizes 1$50 \mathrm{bp}$, there are 183,898 indels in 876,691 TRs (21\%). Our results indicate that at least one-fifth of the TRs in the sequenced individual are heterozygous.

\section{TRs in genes and promoters}

We investigated the intersection of TRs and coding regions, and found 17,800 coding regions in 7,372 genes contained a TR. Of these TRs, 2,094 TRs (12\%) intersect an indel as annotated by either mapped PacBio or Illumina data. These heterozygous TRs within coding regions are found in 1,514 genes (6.5\% of annotated genes).

In addition, we investigated the $2 \mathrm{kbp}$ sequence upstream of annotated genes (Fig. 5). Of the 42,244 TRs identified in these promoter regions, 8,516 (19\%) have an indel annotated based on the union of PacBio and Illumina data.

\section{Discussion}

An improved genome assembly for Atlantic cod

Here we present a new and significantly improved version of the Atlantic cod genome assembly with successful integration of data from different sequencing technologies. The final assembly (gadMor2) was created using a novel reconciliation method, aimed at combining the strengths of four separate assemblies into an integrated assembly maximizing desired metrics, i.e. contig length, scaffold lengths, gene content and accordance with read data (Table 1). The individual assemblies used for the reconciliation were based on different combinations of sequencing technologies and assembly programs, and varied widely in the different studied metrics. Importantly, the inclusion of the long PacBio reads spanning many more repeats than the other sequencing technologies, resulted in an assembly (CA454PB) with a contig N50 an order of magnitude longer than the other assemblies, contributing directly to the long contig N50 of the final assembly. To our knowledge, the specific approach used in generating $\mathrm{CA} 454 \mathrm{~PB}$, where the raw, uncorrected PacBio reads 
Table 5 Overview of tandem repeat statistics

\begin{tabular}{lllll}
\hline Assembly & $\begin{array}{l}\text { Total size } \\
\text { assembly (Mbp) }\end{array}$ & Number of TRs & $\begin{array}{l}\text { Mean length } \pm \text { standard } \\
\text { deviation (bp) }\end{array}$ & $\begin{array}{l}\text { Density of TRs } \\
\text { (\% of assembly) }\end{array}$ \\
\hline gadMor1 & 832 & 970798 & $56.50 \pm 45.17$ & 8.75 \\
ALPILM & 660 & 530801 & $49.64 \pm 53.64$ & 5.41 \\
NEWB454 & 656 & 601043 & $60.35 \pm 62.72$ & 7.01 \\
CA454ILM & 647 & 921184 & $73.43 \pm 97.89$ & 10.2 \\
CA454PB & 682 & 890967 & $86.01 \pm 130.64$ & 10.6 \\
gadMor2 & 643 & 876691 & $84.32 \pm 121.86$ & 10.9
\end{tabular}

were first trimmed and then assembled without correction, together with Illumina and 454 data (see Methods), has not been previously described. A similar approach was used in generating one assembly for Atlantic salmon (see Supplement in [9]), but the sequence in that assembly did not contribute to the final assembly. End-sequenced BAC (Bacterial Artificial Chromosomes) libraries provide long-range information in the $100 \mathrm{kbp}$ range, and such sequences are available for Atlantic cod [5]. The insert size distribution of the BAC-end library was bi-modal (Supplementary Figure 10 in [5]), which is not handled properly in the Celera Assembler. We therefore included these data in the Newbler assembly (NEWB454) only, which contributed to this assembly having the longest N50 scaffold of the original assemblies. The assembly using a combination of 454 and Illumina sequencing reads

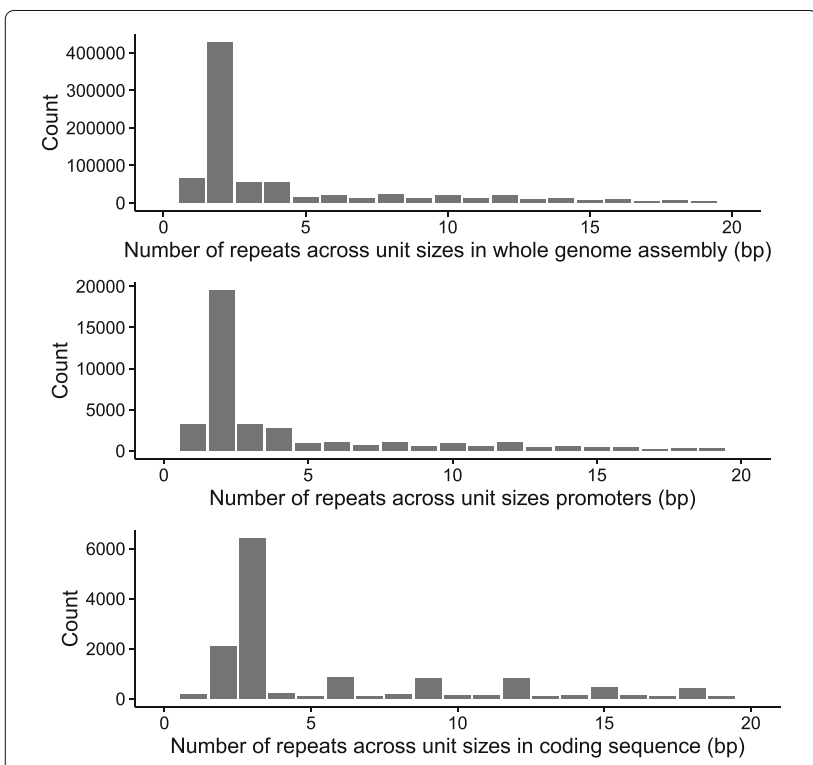

Fig. 4 The number of tandem repeats categorized based on unit size. Only tandem repeats with unit size 1-20 bp are shown. A unit size of one indicates a mononucleotide tandem repeat, two a dinucleotide, three a trinucleotide, repeats etc. The horizontal axis denotes the unit sizes of the repeat, while the vertical axis shows the count of the particular repeat
(CA454ILM) was the most complete in regards to genes as found by the assembly validation tools CEGMA and BUSCO. While the available Illumina sequencing read datasets did not exactly match the recommendations for ALLPATHS-LG [31], the resulting assembly (ALPILM) performed better than gadMor1 with regards to N50 contig and scaffold metrics. Despite its short contigs and scaffolds, this assembly contributed to the assembly reconciliation process, resulting in longer scaffolds. Our results illustrate a dilemma in obtaining high quality genome assemblies: different combinations of datasets and software using algorithms optimized for certain characteristics of the datasets yield assemblies that are of good quality on different combinations of desired quality criteria, but hardly ever on all [30]. Assembly reconciliation helps solve this issue [41], however even our integrated assembly does not rank best on every single metric evaluated. Further improvements in sequencing technology and assembly algorithms are necessary to resolve this problem in genome assembly.

Due to the fragmented nature of the first version of the Atlantic cod genome, gadMor1, gene-models were reconstructed for the annotation using information from the annotated stickleback genome (i.e., ordering and orienting the contigs based on stickleback gene models), and by manual curation (Supplementary Note 17 in [5]). In contrast, the gadMor2 gene models were automatically annotated directly on the genome assembly. This automated annotation did not annotate pseudogenes, in contrast to the manual curated annotation for gadMor1. The difference in annotation might explain why the CEGMA validation results are slightly lower for the new reference genome, since well-annotated gene models in stickleback would be transferred to gadMor1 (Table 1). The gadMor2 assembly shows fewer indications of potential assembly errors as detected by $F R C^{b a m}$ and in comparison to the linkage map, but more according to the REAPR program. This difference is associated with longer contigs and scaffolds in gadMor2, which enabled REAPR to estimate more long-range errors. The predicted transcriptome is larger in gadMor2 (Table 2), although more genes are found with $\mathrm{BUSCO}$ in the gadMor1 predicted transcriptome. $\mathrm{BUSCO}$ 


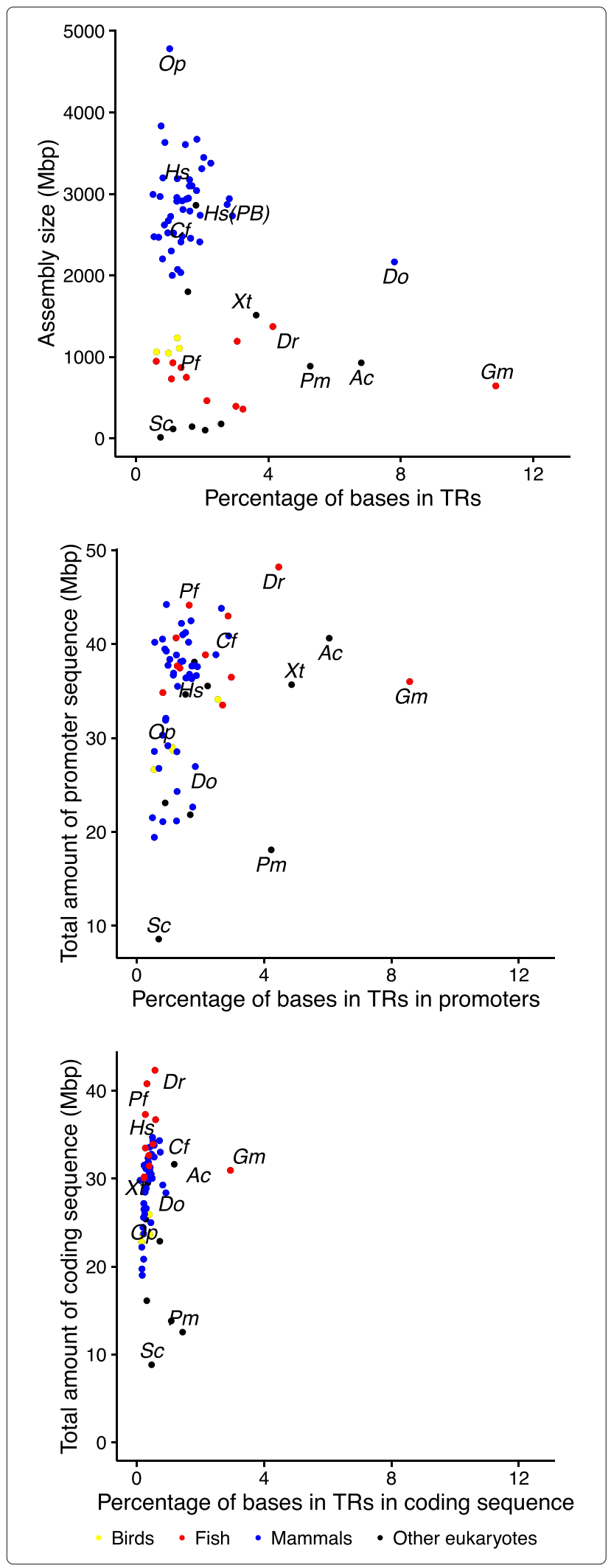

Fig. 5 The density of TRs in genome assemblies, promoters and coding regions. The assemblies shown here are from Ensembl release 81, excluding gadMor1, plus a human genome based on PacBio data, the California sea hare Aplysia californica and gadMor2 $(n=71)$. The panels show the density (percentage of bases) of TRs in the whole assembly, coding regions and promoter regions, respectively. The human PacBio assembly is not included in the gene and promoter analysis because it has no annotation, and the opossum is lacking for technical limitations. The species marked are Oc (Ochotona princeps, pika), Hs (Homo sapiens, human), Hs(PB) (Homo sapiens, human, PacBio based assembly), Cf (Canis familiaris, dog), Do (Dipodomys ordii, kangaroo rat), Xt (Xenopus tropicalis, frog), Pf (Poecilia formosa, Amazon molly), Dr (Danio rerio, zebrafish), Pm (Petromyzon marinus, lamprey), Sc (Saccharomyces cerevisiae, yeast), Ac (Aplysia californica, California sea hare) and Gm (Gadus morhua, Atlantic cod, gadMor2)

is designed to detect genes that are often short (as conserved genes are often short [65]), which means they are more likely put together properly in the gene-model optimized gadMor1 assembly, since longer genes are more likely to be fragmented.

\section{Causes of fragmentation of cod assemblies}

To understand the fragmented nature of gadMor1, we first focused on the rate of heterozygosity, as substantial differences between the homologous chromosomes of diploid organisms can fragment an assembly [66]. We compared the heterozygosity rate of the gadMor2 genome assembly (based on sequencing data obtained from the same individual as gadMor1) to three other fish with genomes for which such data is available, i.e. the miiuy croaker [67], three-spined stickleback [68] and Atlantic herring [69], and to the sea squirt Ciona savignyi [66], a species with extremely high heterozygosity (Table 3 ). The genomes for the fishes have been assembled to high contiguity (Table 3). Although a direct comparison may be confounded by the differences in population structure (in addition to different datasets and programs used [70]), and by a larger uncertainty connected with calling indels correctly than with SNP calls [71], there are substantial differences between the different species. gadMor1 had a N50 contig length of $2.3 \mathrm{kbp}$ (Table 1), substantially shorter than even Ciona savignyi which has an order of magnitude higher SNP rate than Atlantic cod. While species with higher SNP rates seem to have shorter N50 contig length (disregarding cod), the sequencing and assembly strategies for the different organisms vary. For gadMor1, the high SNP rate may have had some impact on the fragmentation (Fig. 6), but it is not the main explanation.

Different combinations of sequencing technology and assemblers vary in their proportion of TRs present in the resulting genome assembly (Fig. 3). Assemblies with higher density in TRs also have more sequence in contigs 


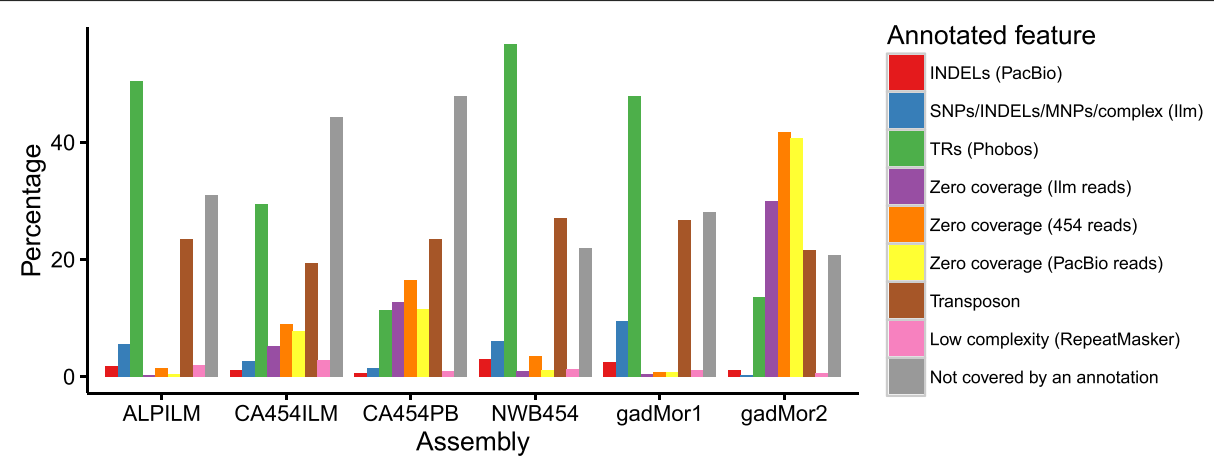

Fig. 6 The intersections between contig termini and different annotated features. The percentage of contig termini (the position of the terminal nucleotides of each contig) intersecting different annotations of the genome

(i.e., less sequence in gaps), indicating that TRs are more completely assembled. The more fragmented assemblies (ALPILM, NEWB454 and gadMor1) have a lower density of TRs and shorter TRs on average, suggesting that TRs led to fragmentation of the assembly (Table 5). Indeed, these assemblies have a much higher proportion $(\geq 40 \%)$ of contig termini intersecting TRs (Fig. 6) than the TR density of $10.9 \%$ in gadMor2 (Table 5). Only CA454PB and (the largely CA454PB derived) gadMor2 have about $10 \%$ of their contig termini intersecting TRs. The remaining gaps in CA454PB and gadMor2 are associated with a lack of sequence coverage and TEs longer than the PacBio read lengths (Fig. 6). This illustrates the importance of the availability of the PacBio reads, which was the only read type able to span the multitude of TRs in the genome. As illustrated in Fig. 2, gadMor2 has a much higher contiguity, while a large fraction of gaps in gadMor1 are flanked with TRs. Thus, our approach to assemble the genome has addressed the fragmentation affecting the gadMor1 assembly. In conclusion, the high occurrence of TRs in the cod genome has caused the fragmentation of gadMor1 and all assemblies except CA454PB and (the largely CA454PB derived) gadMor2. Without the inclusion of reads obtained from the PacBio technology, or similar sequencing technologies that can span long TRs, assembly of genomes with a high density of TRs, such as the Atlantic cod, to a high sequence contiguity will be significantly more challenging.

\section{The Atlantic cod genome reveals an extraordinary high density of TRs}

We have confirmed and extended previous results showing high genomic densities of STRs in Atlantic cod $[61,72]$ by comparison with 68 eukaryote genomes (mostly vertebrates, Fig. 5). While most of the species studied have fewer than $2.5 \%$ of bases in TRs, California sea hare, kangaroo rat and Atlantic cod have more than $6 \%$ bases in TRs. Atlantic cod has by far the highest density (amount of sequence in TRs) and frequency (the rate of TRs, Additional file 1: Figure S1) of TRs in the whole genome assembly, coding regions and promoters, with only California sea hare having a higher frequency (but not density) of TRs in promoter regions.

\section{Potential role of TRs in evolutionary processes in Atlantic cod}

The mutation rates of TRs, and especially STRs, are orders of magnitude higher than those of other genomic sequences $[19,73,74]$. In the sequenced individual, we find that one fifth of the annotated TRs are heterozygous, with somewhat lower proportions in promoters (19\%) and coding regions (12\%). These results are based on the mapping of Illumina and PacBio reads, but are likely underestimates. Most of the TRs in cod have a short repeat unit that mutate by adding or removing at least one repeat unit, for instance, two nucleotides in the case of dinucleotide repeats. Small differences between two long alleles of a TR would likely not be captured by our analyses, because the Illumina reads would not map well to these $[15,75]$, and the PacBio reads might not give sufficient resolution.

In humans, TRs are best known in connection with diseases such as Huntington's Disease [76]. In other species, variability (multiple alleles at a locus within a population) in TRs in promoter regions has been associated with diverse phenomena such as behavior in voles [77] and to skull form in dogs [78]. In both Saccharomyces cerevisiae and humans, some promoter regions contain TRs $[79,80]$, for which variation in length has been linked to variation in expression $[80,81]$. TRs in promoter regions may also contribute to expression divergence in great apes [82] and speciation in primates [83]. There is also variability in TRs in genes leading to functional variation such as in Saccharomyces cerevisiae, where TRs in cell-wall genes underlie variation that causes alterations in phenotype, with different genotypes have differences in adhesion, flocculation or biofilm formation [84]. Further, in Hawaiian mints, 
variation in a gene coding for a flowering time protein is associated with colonization and radiation of the plant, with longer versions of the gene existing in younger populations and this is suggested to contribute to morphological change and speciation [85]. Interestingly, Atlantic cod has a higher frequency of TRs than these species within both promoters and coding regions (Additional file 1: Figure S1).

The sequenced individual was from the North-East Arctic cod population, the largest cod population in the world [86], with a large effective population size [87]. Extrapolating the high mutation rate of TRs, and the observed level of polymorphism in this single individual, suggests that most TRs are polymorphic at a population level. These polymorphic TRs contribute substantially to standing levels of genomic variation in Atlantic cod populations within and in the vicinity of genes.

\section{Conclusions}

Atlantic cod has an extraordinary amount of TRs compared to other species. This repeat content has previously led to complications for assembling the genome. Here, this has been addressed with the usage of the long PacBio sequencing reads and reconciliation of multiple assemblies. The large amount of TRs is likely to have profound evolutionary impact. In particular, the TRs in coding and regulatory regions will drive genetic variation affecting the function or regulation of genes in Atlantic cod populations. It remains to be investigated how cod populations evolve under variable environmental conditions with respect to TRs, and whether selection for repeat variation can lead to rapid evolutionary adaptations.

\section{Methods}

\section{Sequencing}

All read datasets originated from DNA extracted from the same individual fish, designated NEAC_001, a wildcaught male specimen of the North-East Arctic population, sampled with the main purpose for sequencing initiative of the Atlantic cod genome and described in detail in [5]. We always strive to limit the effect of our sampling needs on populations and individuals. This individual was sampled in connection with a research survey conducted by Norwegian Institute for Water Research as part of part of larger hauls for stock assessments. The fish were humanely sacrificed by administration of other sedatives before sampling in accordance with the guidelines set by the 'Norwegian consensus platform for replacement, reduction and refinement of animal experiments' (www.norecopa.no). See Additional file 1: Table S1 for an overview of different DNA datasets generated from this individual.

Roche/454 reads were sequenced as described previously [5]. The Roche/454 software gsRunProcessor version 2.6 was used to redo basecalling for all sequencing runs generated for the NEAC_001 sample [5].

One hundred eighty bp insert size and 300 bp insert size libraries were constructed with Illumina DNA paired end sample preparation reagents and sequenced at the Norwegian Sequencing Centre. The $5 \mathrm{kbp}$ insert size libraries were prepared with the Illumina Mate Pair gDNA reagents and sequenced at the McGill University and Génome Québec Innovation Centre. All Illumina libraries were sequenced on the HiSeq 2000 using V3 chemistry 100 bp paired end reagents.

PacBio SMRT sequencing was performed on PacBio RS instrument (Pacific Biosciences of California Inc., Menlo Park, CA, USA) at the Norwegian Sequencing Centre (www.sequencing.uio.no/) and at Menlo Park. Long insert SMRTbell template libraries were prepared at NSC (10 kbp insert size) and Menlo Park (22 kbp insert size) according to PacBio protocols. In total, 147 SMRT-cells were sequenced using $\mathrm{C} 2$ and XL polymerase binding and $\mathrm{C} 2$ and $\mathrm{XL}$ sequencing kits with $120 \mathrm{~min}$ acquisition. Approximately $7.6 \mathrm{~Gb}$ of library bases were produced from $10 \mathrm{~kb}$ SMRTbell libraries sequenced on 102 SMRT cells using C2/C2 chemistry (average polymerase read length of $3 \mathrm{~kb}$ ). The $22 \mathrm{~kb}$ SMRTbell library was sequenced using C2/XL (22 SMRT cells, average polymerase read length of $4.5 \mathrm{~kb}$ ) and XL/XL (23 SMRT cells, average polymerase read length of $5 \mathrm{~kb}$ ) chemistry producing $5.5 \mathrm{~Gb}$ of library bases.

\section{Assembly}

An overview of the usage of different sequencing data in the different assemblies is in Additional file 1: Table S1.

\section{ALLPATHS-LG assembly, ALPILM}

An ALLPATHS-LG [31] assembly was created using only the Illumina reads. Paired end 100 bp Illumina reads from a 180 bp insert size library were input as fragment reads, while paired end $100 \mathrm{bp}$ reads from a $300 \mathrm{bp}$ insert library and $100 \mathrm{bp}$ reads from a 5k mate pair library were input as jumping reads. Only half of the fragment reads were used in the assembly to have the recommended coverage (Additional file 1: Table S1). The release R48639 of ALLPATHS-LG was used.

\section{Newbler assembly, NEWB454}

Newbler version 3.0 was used to assemble the 454 sequencing data together with BAC-ends previously generated for [5], with the options "-large -het -repfill -sio -info -a 0 ". In contrast to the Newbler assembly done for the first version of the Atlantic cod genome [5], we did not filter out 454 reads consisting entirely of short TRs, as newer versions of the Newbler program are better able to deal with these reads. 
In its output, Newbler gives a file with all scaffolds, including all unscaffolded contigs longer than $2 \mathrm{kbp}$, and a separate file with all contigs, regardless of their inclusion in a scaffold. Using BLAT version 3.5 [88] we mapped the flanking sequences of SNPs in the linkage map (personal communication, Sigbjørn Lien) $(\mathrm{n}=9355)$ to all contigs. For each mapped SNP, the longest contig to which it mapped was added to the primary output, with the rationale that sequences with SNPs should be included in the assembly. The final assembly thus contains all scaffolds, all contigs longer than $2 \mathrm{kbp}$ and the longest unplaced contigs with a mapped SNP.

\section{Celera Assembler assembly based on 454 and Illumina reads, CA454ILM}

Celera Assembler's meryl (SVN snapshot dated 2nd of April 2013) [32] was used to count k-mers in the two paired end Illumina read libraries, of $180 \mathrm{bp}$ and $300 \mathrm{bp}$ insert sizes and of length $100 \mathrm{bp}$.

FLASH version 1.2.3 [89] was used to merge the overlapping reads from the $180 \mathrm{bp}$ library using default options.

The merTrim program, also from Celera Assembler, was used to correct Illumina reads by changing infrequent k-mers to frequent k-mers: starting from the first (last) frequent k-mer in a read, if the next (previous) k-mer is infrequent, then the most recently added base must be an error. To correct it, the three substitution changes are tested; if all k-mers spanning this base are now frequent, the change is accepted. If not, the four insertion and one deletion changes are tested; likewise, if all k-mers spanning this change are now frequent, the change is accepted. Otherwise, the base is left unchanged. Finally, the read is trimmed to the largest region with all $\mathrm{k}$-mers designated as frequent $\mathrm{k}$-mers.

Celera Assembler was used to remove duplicate reads from the $300 \mathrm{bp}$ and $5 \mathrm{kbp}$ Illumina reads libraries with its run runCA-dedupe pipeline.

All 454 reads were converted from .sff files to .fastq and .frg files using Celera Assembler's sffToCA with options "linker flx -linker titanium -insertsize ins_size std_ins_size -trim chop -libraryname lib_name -output output_name", with insert sizes and standard deviations at 1100, 320; 1230, 350; 1440, 440; 1760, 470; 2650, 700; 7000, 1900; 19000,4750 for the different sequencing libraries increasing in insert size (Additional file 1: Table S1). The insert sizes and standard deviations were those reported by Newbler.

The 454 reads were error-corrected using the merTim program, as above, and trimmed as described in Prüfer et al. [90], removing duplicated pairs of reads, error-prone ends of reads, reads with sequence not confirmed by other reads and chimeric reads. Because the insert length distribution of the paired reads from the $20 \mathrm{kbp} 454$ mate pair library showed a bimodal distribution (Additional file 1: Figure S4, in [5]), and since Illumina mate pair libraries contain contamination with pair of reads with the opposite orientation, the scaffolds from this assembly were used to filter out reads from the $20 \mathrm{kbp} 454$ library and the 5k Illumina library by mapping the reads to the scaffolds using BWA-MEM [52], and removing any pair of reads that mapped closer than $10 \mathrm{kbp}$ and $2 \mathrm{kbp}$, respectively.

After the error correction steps, all $5 \mathrm{kbp}$ mate pair Illumina reads, $6 x$ coverage of the 300 bp insert size Illumina reads and $25 \mathrm{x}$ of the merged $180 \mathrm{bp}$ insert size Illumina reads were assembled together with all the 454 reads. Seqtk [91] from November 2012 was used to extract these reads.

The assembly used this spec file (only non-default options shown):

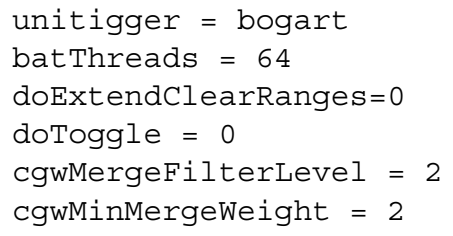

Contigs from Celera Assembler's degenerate contig file, normally excluded from scaffolds, were added to the assembly if they contained flanking sequence from a SNP from the SNP-chip as described above for the Newbler assembly.

\section{Celera Assembler assembly based on PacBio, 454 and Illumina reads, CA454PB}

All processing of Illumina and 454 reads were redone as described above, using Celera Assembler 8.1.

Filtered subreads of PacBio reads were trimmed using Celera Assembler 8.2 alpha with this spec file (only nondefault options shown):

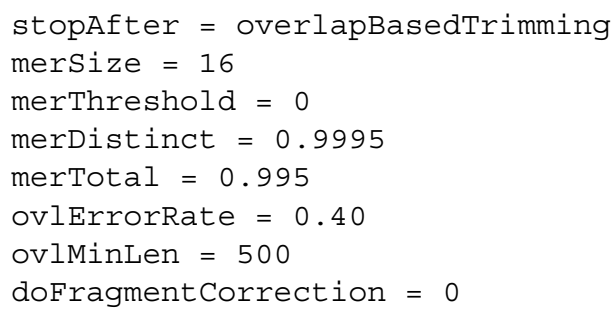

Assembly below was run with this spec file (only nondefault options shown):

mersize $=16$

merThreshold $=0$

merDistinct $=0.9995$

merTotal $=0.995$

doOBT $=0$ 


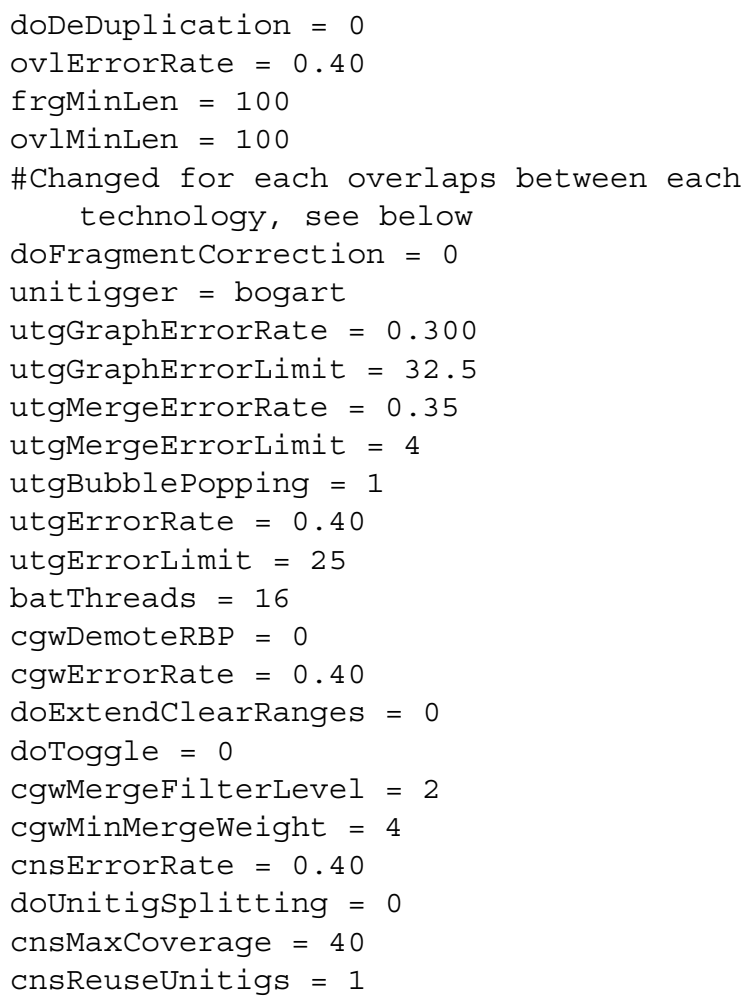

The assembly contains all paired 454 reads, $25 \mathrm{x}$ of merged reads from the $180 \mathrm{bp}$ insert size Illumina library and the trimmed PacBio reads, and was run with Celera Assembler 8.2 alpha. To accommodate vastly different error rates between the Illumina/454 and PacBio reads, overlaps were computed using a different percentage maximum allowed error (inverse of percentage identity) cutoff for each pair of technologies being overlapped. Overlaps between Illumina and 454 reads were computed to a maximum of $6 \%$ error and minimum overlap of $100 \mathrm{bp}$; overlaps between an Illumina/454 read and a PacBio read was computed to a maximum of $20 \%$ error, also with a minimum overlap of $100 \mathrm{bp}$; overlaps between two PacBio reads were computed to a maximum of $40 \%$ error and minimum overlap of $1000 \mathrm{bp}$. For each read end, the bogart unitig construction algorithm will pick the longest overlap and use only those for constructing initial unitigs, similar to the BOG algorithm in [32]. Bogart uses clusters of partially aligned reads (discovered via pre-computed overlaps) to detect junctions between repeat and nonrepeat sequence. If a detected repeat is spanned by either a read or a mate-pair, the repeat is left intact, otherwise, the unitig is split into at least three pieces: one for each side of the repeat, and at least one for the repeat itself.

The rest of the assembly process was run as normal, aside from much higher error rate acceptance at all steps and a non-default selection of unique unitigs. Because PacBio reads confuse Celera Assembler's classification of unique unitigs (which can be used as seeds for creating contigs) and non-unique unitigs (often repeats that could be placed several times in the assembly), we ran the classification tool markRepeatUnique by hand, specifying that unique unitigs could not have a single reads spanning more than $90 \%$ of its length, up to $15 \%$ of the unitig could have a depth of only 3 reads, and must have had at least 200 reads and be at least 10,000 bp long. Command:

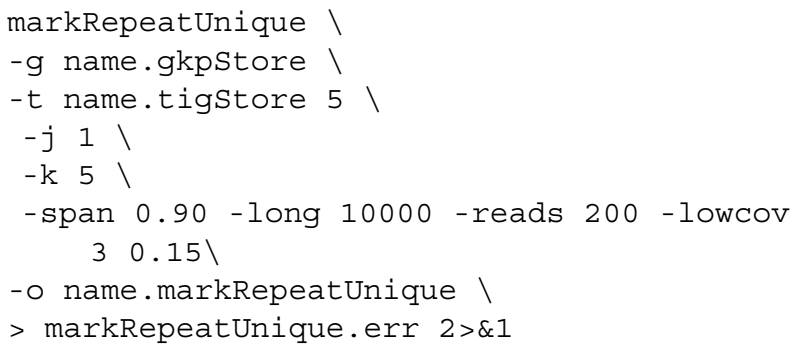

Degenerate sequences that either contained a SNP (as described earlier) or a gene found with CEGMA version 2.4.010312 [38, 65], were added to the assembly output.

\section{Pilon and PBJelly}

All four assemblies described above were processed with PBJelly (SVN snapshot 23rd September 2014) [34], a tool that maps PacBio reads back to the assembly and uses them to close gaps both between and within scaffolds. The content of the configuration file Protocol.xml:

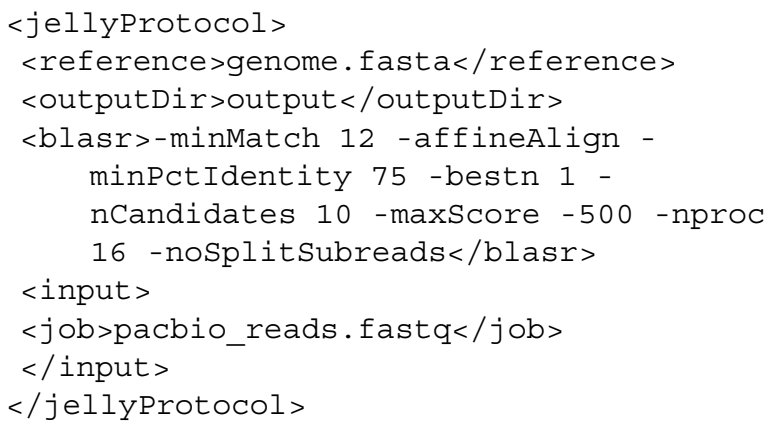

Commands used:

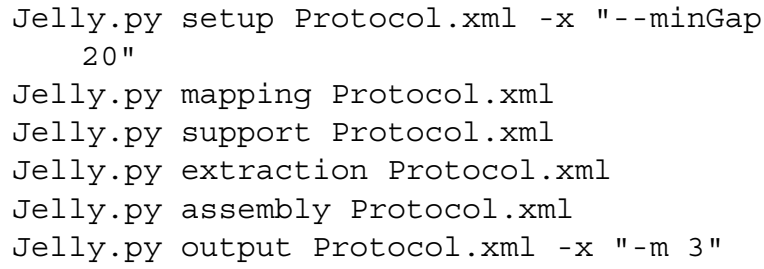

Pilon version 1.9 , a program to automatically improve assemblies [33], was applied to both the original and the PBJelly version of the assemblies, using all 454 reads, the reads from the $300 \mathrm{bp}$ and $5 \mathrm{kbp}$ insert size Illumina libraries, mapped with BWA-MEM 0.7.9a and sorted by samtools 0.1.19 [92]:

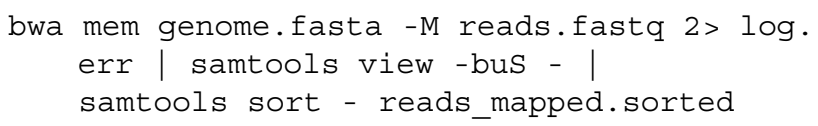


Pilon options were (not showing all the libraries):

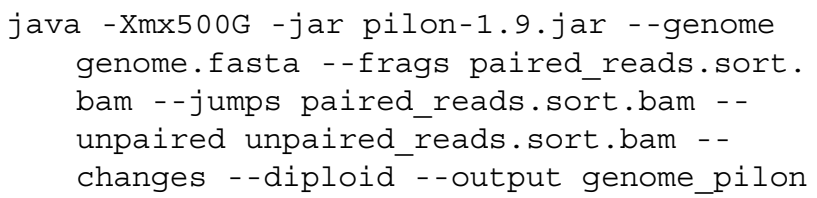

And the reads from all PacBio libraries, mapped with blasr from SMRTanalysis 2.2.0 and sorted by samtools 0.1.19:

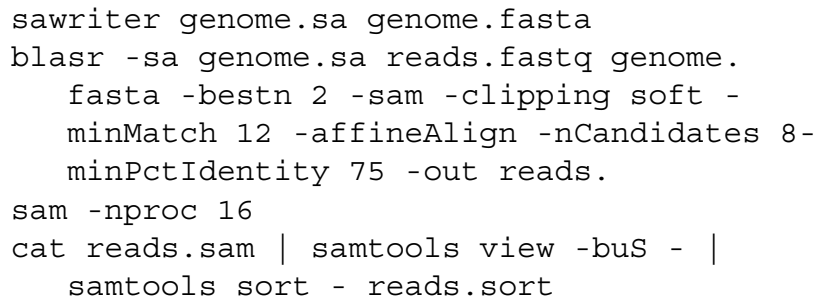

This resulted in four different versions of each assembly: the original; one processed with PBJelly; one processed with Pilon; one and processed with both PBJelly and Pilon. Based on the results of the validation tools against applied to all versions of the assemblies (see below), one version of each assembly was chosen for merging, the versions of ALPILM, NEWB454 and CA454PB after application of both PBJelly and Pilon and the version of CA454ILM after application of Pilon only.

\section{Validation}

To evaluate assembly quality, several validation tools were applied. Both REAPR [36] and $F R C^{b a m}$ [35] use paired Illumina reads to evaluate an assembly, giving a measure of the number of potential errors. Instead of using the raw reads, we used error corrected reads dumped from the ALLPATHS-LG assembly, reducing the running time of both the alignment step and the tools themselves.

Isoblat was used to determine how much of the Newbler transcriptome of 454 and Sanger reads was aligned to the different assemblies [37]. It was run with default options.

CEGMA is a tool that annotates 458 highly conserved genes in an assembly, and it can be used to assess the completeness of the genome assembly $[38,65]$. Version 2.4 was applied to all different versions of the assemblies.

BUSCO is similar to CEGMA in that it assesses the completeness of a genome by trying to find a set of universal single-copy orthologs [39]. In this study, we used the actinopterygii specific set of 3698 genes to investigate the completeness of the assemblies generated here.

A linkage map for Atlantic cod has been created from a set of 9355 SNPs (personal communication, Sigbjørn Lien). We used blat_parse.py to compare the linkage map to different assemblies to evaluate the completeness and long-range correctness. Briefly, this involved mapping the flanking sequences of the SNPs to the assembly using BLAT version 3.5 [88] and options "-noHead -maxIntron=100 genome.fasta flanking_sequences.fasta" and then parsing the output file while comparing with the order of the SNPs in the linkage map. A conflict with the linkage map is defined as a sequence that had SNPs mapped to it belonging to more than one linkage group. Some SNPs mapped equally well to more than one linkage group, and these were excluded since we could not confidently judge which mapping was correct.

\section{Merging of assemblies}

Each assembly was aligned against itself using nucmer [93], and any sequences fully contained in another sequence with more than $98 \%$ identity were removed. Scaffolds were split with a split_asm_lg.py (available on the github repository together with the other scripts mentioned in this section) if they conflicted with the linkage map. A scaffold in conflict is split into three pieces, from the start of the scaffold following one linkage group to the last basepair in the flanking sequence of the last SNP in that linkage group, and from the first basepair in the flanking sequence of the first SNP in another linkage group. The middle piece is not used since we do not know where exactly the transition from linkage group to another happens. Sequences shorter than 1000 bp were removed to better facilitate the whole assembly alignment process.

The four assemblies selected for merging were aligned together using Mugsy. Mugsy uses nucmer from the Mummer package [93] to find similar sequence in different assemblies and subsequently refines the alignment. It outputs a MAF (Multiple Alignment Format) file, consisting of blocks of multiple alignments with information where exactly in the sequences the alignment is (starting at $100 \mathrm{bp}$ and ending at $300 \mathrm{bp}$ in scaffold $\mathrm{X}$ in assembly $\mathrm{Y}$ for instance). The MAF file was parsed by merge_asms.py. Based on the validation criteria described above, one assembly was chosen as the skeleton (CA454ILM), and a second assembly was chosen as the sequence contributing part (CA454PB). The CA454ILM assembly was chosen as skeleton because it was the most complete with regards to genes, and CA454PB was chosen as sequencing contribution assembly was chosen because it had the least gaps. A first pass through the alignment blocks of the first assembly was used to close gaps using the sequences from the CA454PB assembly, or the sequence in each alignment block with the least amount of missing bases. A second pass through the alignment blocks of the first assembly tried to connect scaffolds from the first assembly (CA454ILM) using scaffolds from other assemblies spanning two scaffolds in CA454ILM. Mugsy was run with these options: 


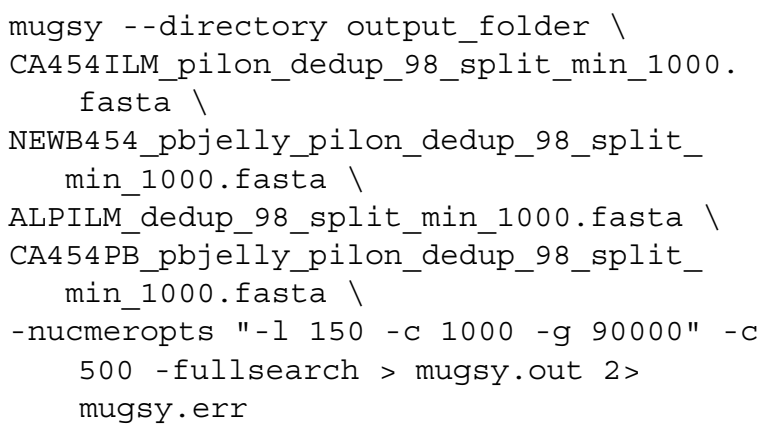

We mapped all paired Illumina and 454 reads to the assembly with BWA-MEM 0.7.9a, and used the scaffold module from SGA [43] to scaffold the merged assembly, increasing N50 scaffold from $850 \mathrm{kbp}$ to $1.15 \mathrm{Mbp}$. Pilon was then applied using all reads excluding PacBio and the 180 bp insert size Illumina library.

\section{Anchoring to linkage map}

Finally, the scaffolds were ordered into linkage groups based on linkage data (personal communication, Sigbjørn Lien) with 100 Ns between two adjacent scaffolds using order_orient_scaffolds.py. Scaffolds with only one SNP kept their existing orientation, while scaffolds with more than one SNP were reverse complemented if more than half the SNPs suggested this. The numbering of the linkage groups is according to Hubert et al. [94].

\section{Transcriptome assemblies}

We obtained transcriptome datasets from three different sequencing technologies, Illumina, 454 and PacBio, from a variety of tissues and different stages. Three different transcriptome assemblies were created: (i) based on assembly of the Illumina reads using Trinity [95]; (ii) assembly of the 454 reads using Newbler [96]; and (iii) clustering the long full-isoform PacBio reads using SMRT-Analysis [97].

\section{Trinity with Illumina reads}

RNA-seq sequencing data used in Penglase et al. [98] (from larvae at different stages and feeding regimes) were obtained from the Sequence Read Archive (SRA) at NCBI with accession ID SRP056073, and adapters and all bases with less than 20 in Phred quality score were removed with cutadapt 1.5 [99]. Trinity version r20140717 [95, $100]$ was run with the normalize_reads option turned on. 654,948 transcripts were assembled. Abundance estimates commands:

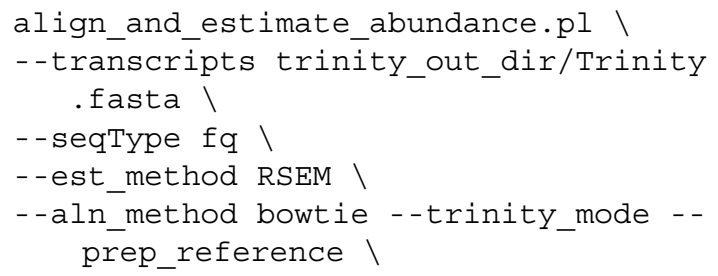

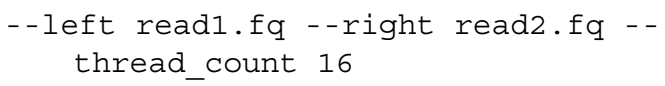

The script filter_fasta_by_rsem_values.pl distributed with Trinity was used to filter the transcript assembly based on abundance, where only transcripts with fragments per kilobase of transcript per million mapped reads (FPKM) of at least 0.05 , and a transcript abundance of at least $1 \%$ of the parent gene's abundance were kept, resulting in 59,379 transcripts.

\section{Newbler with 454 and Sanger reads}

The transcriptome 454 and Sanger reads used in Star et al. [5] (the different tissues listed in Supplementary Table 2 in [5]) were combined with Sanger reads from Kleppe et al. [101], and assembled with Newbler 3.0 using the options -cdna and -vt with these primer sequences:

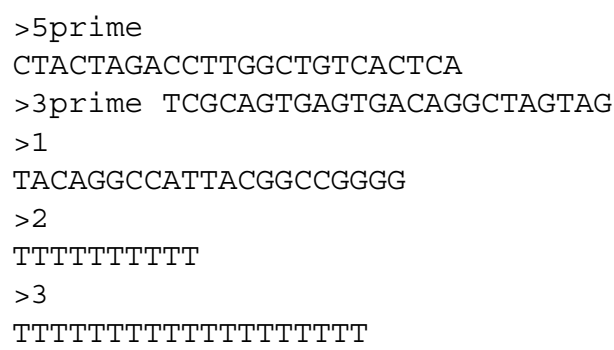

The assembly resulted in 79,025 transcripts.

\section{IsoSeq on PacBio reads}

Equal amounts of RNA were isolated from pools of unfertilized eggs and at 20, 30, 45, 60 and 90 days post hatch. Isolations were pooled and three size-selected fractions based on agarose gel-electrophoresis of RNA were created at sizes 1-2 kbp, 2-3 kbp and 3-6 kbp and sequenced on the Pacific Biosciences RS using P6v2-C4 chemistry [97]. Using SMRT Portal, reads-of-insert were first created for each fraction, and isoform prediction and polishing by Quiver were performed according to the manufacturer's instructions. For the fraction 1-2 kbp, 10,738 high quality isoforms were predicted $(\geq 99 \%$ accurate sequence according to Quiver) and 2,952 low quality ( $<99 \%$ accurate sequence), for the $2-3 \mathrm{kbp}$ fraction 15,688 high quality and 6,898 low quality and for the 3-6 kbp fraction 13,400 high quality and 12,716 low quality transcripts. These 62,392 transcripts were merged into one fasta file and used in further analyses.

\section{Annotation \\ Repeat libraries}

A repeat library for MAKER gene annotation (see below) was created by running RepeatModeler [56] version 1.0.8 on the finished genome assembly with default options.

We also created a repeat library specifically for annotation of transposable elements (https://github.com/uiocels/Repeats). First, RepeatModeler [56] version 1.0.8 was 
run on only the scaffolds longer than N50. LTRharvest [57] and LTRdigest [58], both parts of genometools (version 1.5.7), were used to detect LTR retrotransposons and TRIMs. LTRharvest found LTR retrotransposons with LTRs larger than $100 \mathrm{nt}$, smaller than $6000 \mathrm{nt}$ and with 1500 to 25000 nt between, with a target site duplication (TSD) length of 5 nt. TRIMs were detected by lowering the LTR length requirements to a minimum of 70 nt and a maximum of $500 \mathrm{nt}$ with maximum $1500 \mathrm{nt}$ of internal sequence. Harvested putative LTR retrotransposons were filtered using LTRdigest, which checked for tRNA binding sites. In addition, LTRdigest used Hidden Markov Model (HMM) profiles to identify retrotransposon enzymes (from the GyDB HMM profile collection of retrotransposon specific enzymes [102]). Elements without both tRNA binding sites and a retrotransposon specific enzyme were discarded.

We used scripts provided by Ning Jiang, Megan Bowman and Kevin Childs (Michigan State University) to perform the next analyses $[49,103]$. Only elements containing primer binding sites (PBS) and/or a polypurine tract (PPT) were kept, and only if at least half of the PBS or PPT sequence was located in the internal regions of the putative element and the distance between the LTRs and the PPT/PBS sequence was less than $20 \mathrm{bp}$. Elements that passed this filtering were subjugated to further filtering where sequences with gaps of $\geq 50 \mathrm{nt}$ were discarded. MUSCLE version 3.8.31 [104] was used to align flanking sequences, and elements with $\geq 60 \%$ similarity in flanking sequences was excluded.

Nested LTR retrotransposons were detected by using RepeatMasker with the left LTR sequences of the putative elements and a library of transposases (from a curated library included in the software TEseeker v1.04 [105]). Consensus sequences were produced after all vs. all comparisons using BLASTN. Finally, no elements from different families shared $80 \%$ sequence over $90 \%$ of their length.

RepeatClassifier, which is a program included with RepeatModeler, was used to classify the elements. As many LTR retrotransposons and TRIMs contain TRs in their long terminal repeats, RepeatClassifier classified some elements as being TRs. These elements were renamed to being LTR retrotransposons or TRIMs, while those that were classified into specific LTR families kept their new classification. TransposonPSI [100] was also run. TransposonPSI uses PSI-BLAST to detect distant homology between genomic sequences and a TE library bundled with the program. Contrary to the other programs, TransposonPSI does not output the consensus sequences of elements detected, which made it necessary to perform an additional clustering step. The output sequences were clustered using CD-HIT-EST 4.6.4 [106] with a similarity cutoff of $80 \%$. The relative high amount of dinucleotide repeats in the Atlantic cod genome assembly, led to a large fraction of sequence being labeled as transposons of the CACTA superfamily, as the CACTA representative in the TransposonPSI library contained a TR that spurred false alignments. Thus, elements were only named CACTA if two sources agreed in the classification, the other source being the results of a BLASTX search against the repeat peptide database provided with RepeatMasker (version 4.0.6).

As the detection tools might detect repetitive non-TE genes such as gene families, the sequences were checked for alignments (using BLASTX) with sequences in the curated protein database of UniProtKB/SwissProt [107], which was downloaded November 20th 2015. Sequences were also checked against the repeat peptide database that comes with distributions of the RepeatMasker software. Sequences with matches in the UniProtKB/SwissProt database, but not in the repeat peptide database were discarded. The BLASTX search against repeat peptides in the database also served to classify some of the unclassified elements.

Some sequences remained unclassified, and a collection of HMM profiles was downloaded from the Dfam database (Dfam.org) and HMMER3 was run using the nhmmer module. This further classified some elements into LTR retrotransposons, LINEs, SINEs or DNA transposons. The de novo library was merged with known eukaryotic repeat sequences from RepBase [60] (version 20150807) and served as input for RepeatMasker.

\section{Annotation with MAKER}

MAKER is an annotation pipeline designed to combine the consolidated output from different $a b$ initio gene finders and physical evidence (e.g. protein and RNA-seq alignments) into a set of quality scored gene models (AED score) $[49,50,108]$.

A two-pass iteration with MAKER version 2.31.8 $[49,50]$ was performed on the final genome assembly as described in [109] and in Campbell et al. [110]. First, two $a b$ initio gene finders were trained, SNAP version 20131129 [111] on the genes found by CEGMA version 2.4.010312, and GeneMark-ES version 2.3e [112] on the genome assembly itself. SwissProt/UniProtKB [107] was downloaded 9th of May 2015 (release 2015_04). MAKER was configured to use the two trained ab initio gene finders, the SwissProt/UniProtKB protein database [107], the RepeatModeler repeat library and three different transcriptomes, one based on 454 and Sanger data, one based on Illumina and one based on PacBio. Additional options were these:

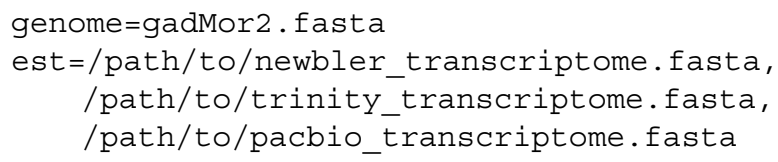




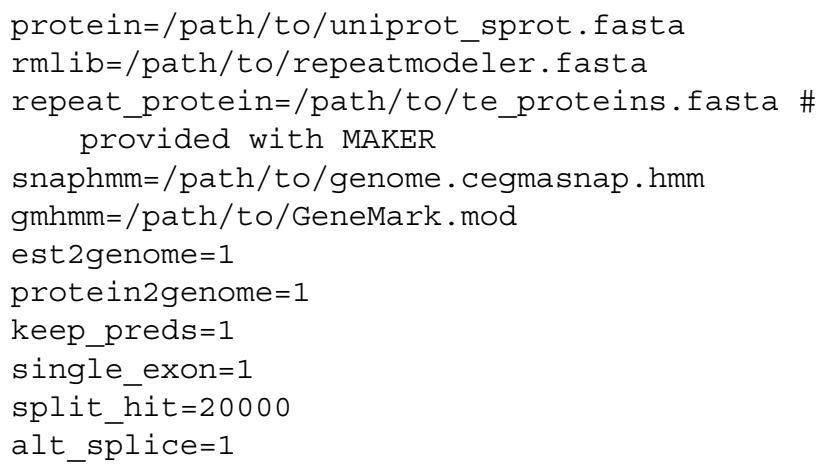

The GFF output from the first pass with MAKER was used to retrain SNAP, and to train AUGUSTUS version 3.0.2 [113, 114] with the PacBio transcriptome. A second pass with MAKER was run with the retrained SNAP, the trained AUGUSTUS and the similar set of input as above, and with these other options:

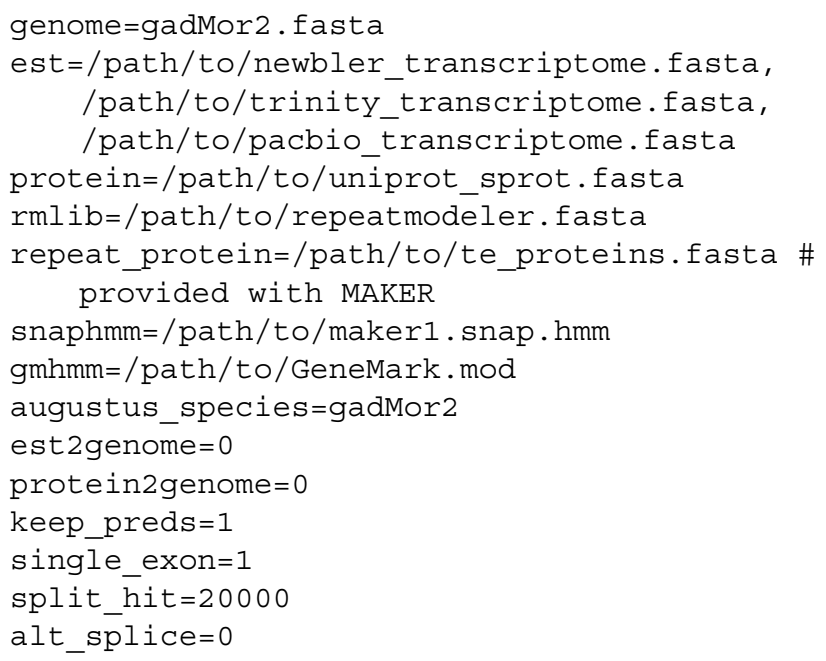

InterProScan version 5.4-47 [115] was run on the protein output of MAKER, providing gene ontologies and classifying protein domains and families. The protein output was BLASTed against SwissProt/UniProtKB release 2015_12, identifying putative gene names, with these options:

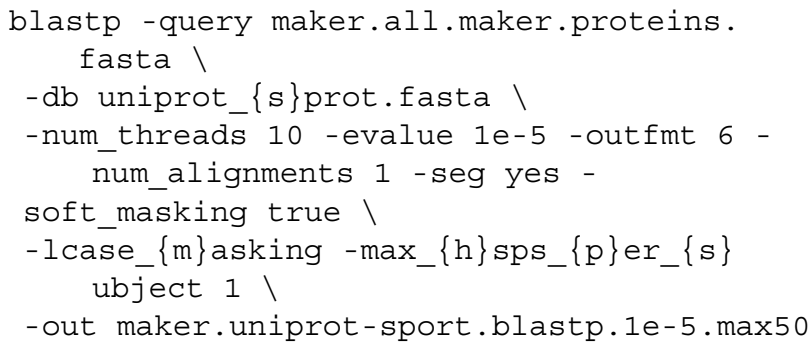

The final gene models set was filtered based on the AED score [116], including only gene models with an AED of less than 0.5. An AED of 0.0 would indicate that the gene model is in perfect accordance with its evidence (mRNA,
EST, protein alignments) and 1.0 that it is not supported by any evidence.

\section{Investigating heterozygosity}

To investigate the heterozygosity of this individual of Atlantic cod, we mapped the 300 bp insert size Illumina sequencing library to the genome assembly using bwa mem version 0.7.9a with the $-\mathrm{M}$ option [52]. Samtools version 1.1 was used to sort the bam files.

bwa mem genome.fasta $-M$ reads.fastq $2>$ log.

err | samtools view -bus - |

samtools sort - reads mapped.sorted

SNP and indel calling was done on the merged bam file using FreeBayes version v0.9.14-17-g7696787 [53], and SNP and indel calls with a quality $>20$ were kept with 'vcffilter -f "QUAL > 20"'. Vcfstats was run on the resulting VCF file, giving the number of SNPs, MNPs, indels and complex regions.

We also mapped all PacBio reads using blasr from SMRT-Analysis 2.3.0, and called indels using PBHoney version $\mathrm{r} 99$ [55], annotating all indels larger than $20 \mathrm{bp}$. This numbered 70,278.

\section{Genome-wide short TR analysis}

TRs of unit size 1-50 bp were detected with Phobos version 3.3.12 [18], options set were "-s 12 -outputFormat 0 $-\mathrm{U} 50$ ", i.e. requiring a minimum score of 12 for each TR, that is, the TR needed a score above 12, i.e. at least 13 mononucleotides, 7 dinucleotide, 5 trinucleotide repeat units, that is, minimum lengths of 13,14 and $15 \mathrm{bp}$, respectively; Phobos native format as output; and up to a motif, or unit, size of $50 \mathrm{bp}$. A range of 1-50 bp was chosen in accordance with Mayer et al. [18]. A config file was then provided for the sat-stat version 1.3.12 program, yielding a diverse output of file with different statistics and a gff file:

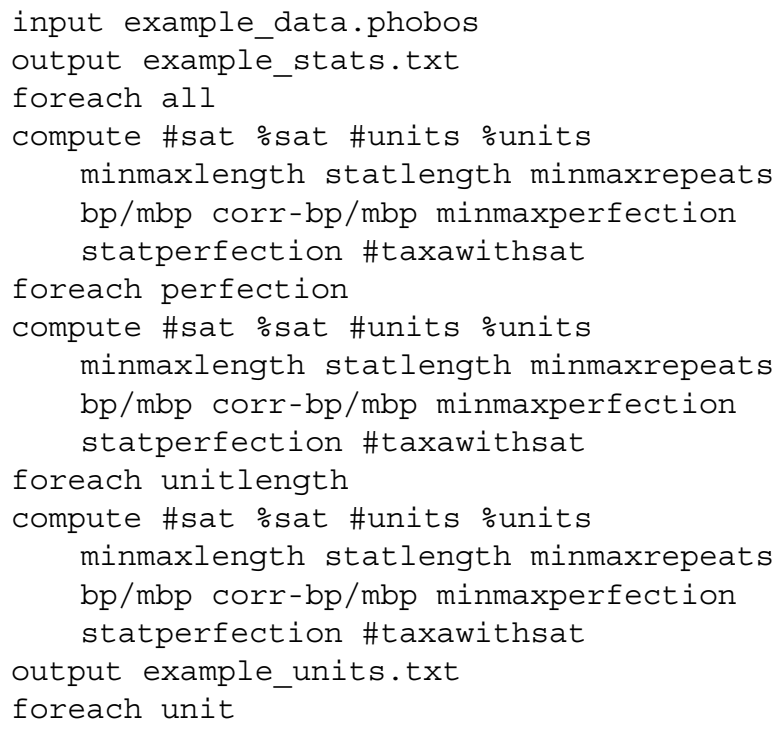


compute minmaxunitlength \#sat \%sat minmaxlength statlength minmaxrepeats $\mathrm{bp} / \mathrm{mbp}$ corr $-\mathrm{bp} / \mathrm{mbp}$

output example_units10.txt

\# Show only those table rows with at least 10 tandem repeats

foreach unit 10

compute minmaxunitlength \#sat osat minmaxlength statlength minmaxrepeats $\mathrm{bp} / \mathrm{mbp}$ corr-bp/mbp

output example_units20.txt

foreach unit 20

compute minmaxunitlength \#sat \%sat minmaxlength statlength minmaxrepeats $\mathrm{bp} / \mathrm{mbp}$ corr-bp/mbp

output example_taxa.txt

foreach taxon

compute \#sat \%sat \#units \%units minmaxlength statlength minmaxrepeats $\mathrm{bp} / \mathrm{mbp} \mathrm{rbp} / \mathrm{mbp}$

compute taxoncontent

compute units\&freq

output example_dist.txt

foreach all

compute \#sat minmaxdistances statdistances corr-bp/mbp

foreach unitlength

compute \#sat minmaxdistances statdistances corr $-\mathrm{bp} / \mathrm{mbp}$

foreach unit

compute \#sat minmaxdistances statdistances corr $-\mathrm{bp} / \mathrm{mbp}$

output example satdistances.txt

foreach all

compute satdistances

foreach unitlength

compute satdistances

foreach unit

compute satdistances

output example-out.gff

print-gff

exit

In addition, STRs were detected with lobSTR 4.0. First, TRF version $4.07 \mathrm{~b}$ was run on the genome assembly with these options "gadMor2.fasta 2778010246 -f - $d$-h", and the resulting gadMor2.fasta.2.7.7.80.10.24.6.dat file was converted to bed format with convert_trf_bed_lobstr.py. A lobSTR index was created with the bed file and the genome, and allelotype classified the STRs using the Illumina 300PE library previously mapped with BWA, using these options:

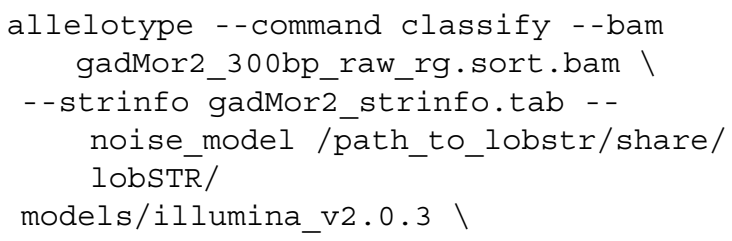

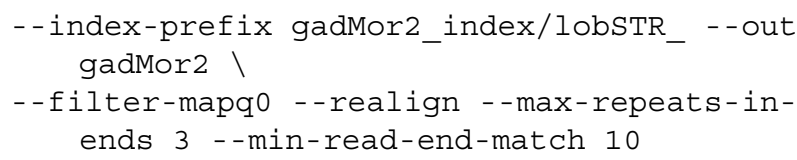

In addition to the different cod assemblies analyzed, we downloaded all assemblies from Ensembl release 81 ( $n=$ 68) (including Atlantic cod) and the California sea hare.

Star et al. [5] released three different assemblies, based on Newbler, Celera Assembler and a gene-model optimized, annotated version of the Newbler assembly, which is the one available from Ensembl and indicated herein as gadMor1. In gadMor1, contigs were reordered according to stickleback proteins during annotation, which resulted in significant improvements in regards to gene model construction compared with the original assembly. In all comparisons between different cod assemblies performed for this work, we compared to the gadMor1 assembly, since it is annotated and likely the one most used.

\section{Contig terminus analysis}

Contigs from the assemblies of ALPILM, NEWB454, CA454PB, CA454ILM, gadMor1 and gadMor2 were created with the "cutN -n 1" command from seqtk version 1.0-r75, which cut at each gap (of at least one basepair, i.e. one or more Ns). The contigs were mapped against the gadMor2 assembly with BWA 0.7.12 and get_positions_non_soft_hard_clip.py was used to create a BED file with only the edges of contigs that map uniquely with a mapping quality of 3 or more.

The intersect option from bedtools version 2.24.0 [117] was used to find overlaps between the contig termini and indels based on PBHoney tails output, SNPs, indels, MNPs and complex regions from mapping Illumina reads (300 bp insert size) to the genome, TRs called by Phobos, lack of coverage by Illumina, 454 and PacBio reads (zero depth as determined by mapped reads and bedtools genomecov), TEs and low complexity regions from RepeatMasker.

\section{Heterozygous TRs}

We used bedtools [117] 2.24.0 to find the intersecting between the indels called by FreeBayes and PBHoney, and the TRs as annotated by Phobos. Indels were filtered based on depth (at least 5 reads) and genotype (0/1, heterozygous).

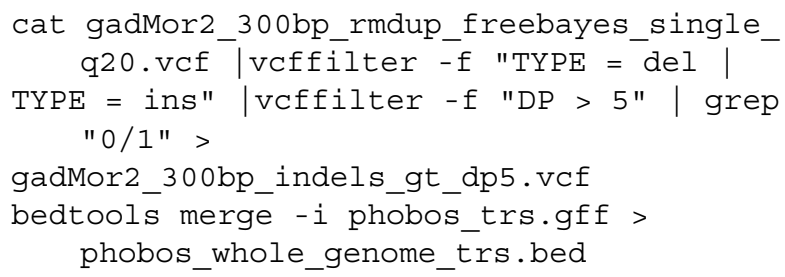


bedtools merge -i gadMor2 honey.bed > pacbio_indels.bed

bedtools merge -i gadMor2_300bp_indels_gt_dp5.vcf > ilm_indels_gt_dp5.bed

bedtools intersect -a phobos_whole_genome_trs.bed -b pacbio_indels.bed > whole_genome_with_trs_pacbio_indels.bed bedtools intersect -a phobos whole genome trs.bed -b ilm_indels_gt_dp5.bed > whole_genome_with_trs_ilm_indels_gt_dp5 .bed

cat whole genome with trs pacbio indels.bed whole_genome_with_trs_ilm_indels_gt_dp5 .bed | sort -k1,1-k2,2n |bedtools

merge >

whole_genome_with_pbilm_indels_gt_dp5. bed

In the annotation of Atlantic cod, some genes were annotated that consist predominantly of TRs. Since these were in the annotation, they have some evidence in the form of protein or transcriptome alignment, and have an open reading frame. However, they seem to have no significant similarity with proteins from SwissProt/UniProtKB, and were removed based on this. This left 19,035 genes for this particular analysis.

cat gadMor2_maker.putative_function. domain_added.aed_0.5.gff |awk '\{if (\$3 $==$ "gene") print $\$ 0\}^{\prime}$ | sort $-\mathrm{k} 1,1-\mathrm{k} 4$, $4 n>$ genes.gff

grep -v unknown genes.gff > known_genes.gff bedtools flank - $i$ known_genes.gff -g gadMor2.fasta.fai $-\overline{1} 2000-r \quad 0-s$ |sort $-\mathrm{k} 1,1-\mathrm{k} 4,4 \mathrm{n}>$ genes. $2 \mathrm{~kb}$.promotors.gff

bedtools intersect -a genes. $2 \mathrm{~kb}$.promotors. gff -b phobos_trs.gff | sort -k1,1 -k4,4 $\mathrm{n}>$ intersect_2kb_promotor_trs.gff bedtools merge $-i$ intersect_2k̄b_promotor trs.gff >intersect_2kb_promotor_trs.bed bedtools intersect -a intersect $2 \mathrm{~kb}$ promotor_trs.bed -b pacbio_indels.bed > promoters_with_trs_pacbio_indels.bed bedtools intersect -a intersect_2kb promotor_trs.bed -b ilm_indels_gt_dp5. bed > promoters_with_trs_ilm_indels_gt dp5.bed

cat promoters_with_trs_pacbio_indels.bed promoters_with_trs_ilm_indels_gt_dp5. bed | sort - $\mathrm{k} 1, \overline{1}-\mathrm{k} \overline{2}, 2 \mathrm{n}$ | bedtools merge > promoters_with_pbilm_indels_gt dp5 . bed

cat gadMor2_maker.putative_function. domain_added.aed_0.5.gff |awk'\{if (\$3
$==" \mathrm{CDS} ")$ print $\$ 0\}^{\prime} \mid$ sort $-\mathrm{k} 1,1-\mathrm{k} 4,4 \mathrm{n}$

$>$ cds.gff

bedtools intersect -a cds.gff -b

known_genes.gff | sort -k1,1-k4,4n >

cds known genes.gff

bedtools intersect -a cds_known_genes.gff b phobos_whole_genome_trs.bed | sort -k1, $1-k 4,4 n>$ cds_with_trs.gff

bedtools merge - $i$ cds_with_trs.gff > cds_with_trs.bed

bedtools intersect -a known_genes.gff - b cds_with_trs.bed >

known_genes_cds_trs.gff

bedtools merge -i cds_with_trs.gff > cds with trs.bed

bedtools intersect - a known_genes.gff -b cds_with_trs.bed > known_genes_cds_trs.gff

bedtools intersect - a cds with trs.bed -b pacbio_indels.bed > cōs_with_trs_ pacbio_indels.bed

bedtools intersect -a cds_with_trs.bed -b ilm_indels_gt_dp5.bed > cds with trs ilm indels gt dp5.bed

cat cds_with_trs_ilm_indels_gt dp5.bed cds_with_trs_pacbio_indels.bed | sort $-\mathrm{k} 1,1-\mathrm{k} 2,2 \mathrm{n}>$ cds_with_trs_ilm_pb_indels_gt_dp5.bed

bedtools merge - $\bar{i}$ cds_with trs ilm pb indels_gt dp5.bed > cds_with_trs_ilm_pb_indels_merged_gt_dp5. bed

bedtools intersect - a known genes.gff - b cds_with_trs_ilm_pb_indels_gt_dp5. bed > cds s_known_genes_intersect_trs_ indel_ilm_pb_gt_dp5.gf $f$

bedtools version 2.24.0 [117] was used to find the intersection between the coding sequence of genes with similarity to proteins from SwissProt/UniProtKB and TRs from Phobos. The result from this was intersected with indels called by FreeBayes (Illumina reads) and PBHoney (PacBio reads).

\section{Additional file}

Additional file 1: Supplementary figures and tables. (PDF $647 \mathrm{~kb}$ )

\section{Acknowledgements}

All computational work was performed on the Abel Supercomputing Cluster (Norwegian metacenter for High Performance Computing (NOTUR) and the University of Oslo) operated by the Research Computing Services group at USIT, the University of Oslo IT-department (http://www.hpc.uio.no/).

Sequencing library creation and high throughput sequencing was carried out at the Norwegian Sequencing Centre (NSC), University of Oslo, Norway; McGill University and Genome Quebec Innovation Centre, Canada and Pacific

Biosciences of California Inc., Menlo Park. We are grateful for the willingness of Helle Tessand Baalsrud, Martin Malmstrøm and Monica H. Solbakken to

investigate their favorite genes as a validation in different draft assemblies. Geir 
Kjetil Sandve provided essential help with implementing different algorithms for assembly reconciliation. We thank Jonas Korlach for his contribution to strategic aspects of the project and commenting on earlier versions of the manuscript, and Cassandra Trier for critical reading of the manuscript.

\section{Funding}

Research Council of Norway project number 199806 to KSJ.

\section{Availability of data and materials}

Accession numbers at ENA (http://www.ebi.ac.uk/ena) for the gadMor2 assembly: WGS: CAEA020000001-CAEA020024862. Scaffolds: LN836027LN845721. Chromosomes: LN845748-LN845770. Accession numbers for the Illumina libraries used for genome assembly: ERX1622640-ERX1622647. Accession numbers for the 454 libraries used for genome assembly: ERX1761596-ERX1761760. Accession numbers for the PacBio libraries used for genome assembly: ERX1787826-ERX1787972. Accession number for the PacBio Iso-Seq used for genome annotation: PRJEB18628. The four original assemblies and gadMor2 available at Figshare: doi.org/10.6084/m9.figshare.3408247. All scripts used for analysis are available at https://github.com/uio-cels/cod2_ scripts and https://github.com/uio-cels/Repeats.

\section{Authors' contributions}

OKT and AJN performed the genome assemblies. OKT and WBR annotated the genome assembly. OKT carried out the TR analysis. JRM, BPW and JK designed and wrote the assembly algorithms. JME, PP, AT-K and MS performed library creation and sequencing. HG and SL created the linkage map. RBE contributed samples for sequencing and evaluated assemblies. OKT, BS, SJ, BPW, KSJ and AJN wrote the paper with help from all the authors. All authors read and approved the final manuscript.

\section{Competing interests}

Jenny M. Ekholm and Paul Peluso are full-time employees at Pacific Biosciences, a company developing single molecule sequencing technologies.

\section{Consent for publication}

Not applicable.

\section{Ethics approval and consent to participate}

\section{Not applicable.}

\section{Author details}

${ }^{1}$ Centre for Ecological and Evolutionary Synthesis, Department of Biosciences, University of Oslo, NO-0316 Oslo, Norway. ${ }^{2}$ Department of Natural Sciences, University of Agder, NO-4604 Kristiansand, Norway. ${ }^{3}$ Centre for Integrative Genetics (CIGENE), Department of Animal and Aquacultural Sciences, Norwegian University of Life Sciences, NO-1432 Ås, Norway. ${ }^{4}$ J. Craig Venter Institute, 9704 Medical Center Drive, 20850 Rockville, MD, USA. ${ }^{5}$ Genome Informatics Section, Computational and Statistical Genomics Branch, National Human Genome Research Institute, National Institutes of Health, 20892 Bethesda MD, USA. ${ }^{6}$ Yale School of Medicine, Yale University, $06520 \mathrm{New}$ Haven, CT, USA. ${ }^{7}$ Pacific Biosciences, Menlo Park CA, USA. ${ }^{8}$ Institute of Marine Research, Nordnes, NO-5817 Bergen, Norway. ${ }^{9}$ Biomedical Informatics Research Group, Department of Informatics, University of Oslo, NO-0316 Oslo, Norway.
\end{abstract}

Received: 27 July 2016 Accepted: 20 December 2016

Published online: 18 January 2017

\section{References}

1. Ekblom R, Wolf JBW. A field guide to whole-genome sequencing, assembly and annotation. Evol Appl. 2014;7(9):1026-43.

2. Li R, Fan W, Tian G, Zhu H, He L, Cai J, Huang Q, Cai Q, Li B, Bai Y, Zhang Z, Zhang Y, Wang W, Li J, Wei F, Li H, Jian M, Li J, Zhang Z, Nielsen R, Li D, Gu W, Yang Z, Xuan Z, Ryder OA, Leung FC-C, Zhou Y, Cao J, Sun X, Fu Y, Fang X, Guo X, Wang B, Hou R, Shen F, Mu B, Ni P, Lin R, Qian W, Wang G, Yu C, Nie W, Wang J, Wu Z, Liang H, Min J, Wu Q, Cheng S, Ruan J, Wang M, Shi Z, Wen M, Liu B, Ren X, Zheng H, Dong D, Cook K, Shan G, Zhang H, Kosiol C, Xie X, Lu Z, Zheng H, Li Y, Steiner CC, Lam TT-Y, Lin S, Zhang Q, Li G, Tian J, Gong T, Liu H, Zhang D, Fang L, Ye C, Zhang J, Hu W, Xu A, Ren Y, Zhang G, Bruford MW, Li Q, Ma L, Guo Y, An N, Hu Y, Zheng Y, Shi Y, Li Z, Liu Q, Chen Y, Zhao J,
Qu N, Zhao S, Tian F, Wang X, Wang $H$, Xu L, Liu X, Vinar T, Wang Y, Lam TW, Yiu SM, Liu S, Zhang H, Li D, Huang Y, Wang X, Yang G, Jiang Z, Wang J, Qin N, Li L, Li J, Bolund L, Kristiansen K, Wong GK-S, Olson M, Zhang X, Li S, Yang H, Wang J, Wang J. The sequence and de novo assembly of the giant panda genome, Nature. 2010;463(7279):311-7.

3. Dalloul RA, Long JA, Zimin AV, Aslam L, Beal K, Blomberg LA, Bouffard P, Burt DW, Crasta O, Crooijmans RPMA, Cooper K, Coulombe RA, De S, Delany ME, Dodgson JB, Dong JJ, Evans C, Frederickson KM, Flicek P, Florea L, Folkerts O, Groenen MAM, Harkins TT, Herrero J, Hoffmann S, Megens HJ, Jiang A, de Jong P, Kaiser P, Kim H, Kim KW, Kim S, Langenberger D, Lee MK, Lee T, Mane S, Marcais G, Marz M, McElroy AP, Modise T, Nefedov M, Notredame C, Paton IR, Payne WS, Pertea G, Prickett D, Puiu D, Qioa D, Raineri E, Ruffier M, Salzberg SL, Schatz MC, Scheuring C, Schmidt CJ, Schroeder S, Searle SMJ, Smith EJ, Smith J, Sonstegard TS, Stadler PF, Tafer H, Tu ZJ, Van Tassell CP, Vilella AJ, Williams KP, Yorke JA, Zhang L, Zhang HB, Zhang X, Zhang Y, Reed KM. Multi-platform next-generation sequencing of the domestic turkey (Meleagris gallopavo): Genome assembly and analysis. PLoS Biol. 2010;8(9):1000475.

4. Shulaev V, Sargent DJ, Crowhurst RN, Mockler TC, Folkerts O, Delcher AL, Jaiswal P, Mockaitis K, Liston A, Mane SP, Burns P, Davis TM, Slovin JP, Bassil N, Hellens RP, Evans C, Harkins T, Kodira C, Desany B, Crasta OR, Jensen RV, Allan AC, Michael TP, Setubal JC, Celton JM, Rees DJG, Williams KP, Holt SH, Rojas JJR, Chatterjee M, Liu B, Silva H, Meisel L, Adato A, Filichkin SA, Troggio M, Viola R, Ashman TL, Wang $H$, Dharmawardhana P, Elser J, Raja R, Priest HD, Bryant Jr DW, Fox SE, Givan SA, Wilhelm LJ, Naithani S, Christoffels A, Salama DY, Carter J, Girona EL, Zdepski A, Wang W, Kerstetter RA, Schwab W, Korban SS, Davik J, Monfort A, Denoyes-Rothan B, Arus P, Mittler R, Flinn B, Aharoni A, Bennetzen JL, Salzberg SL, Dickerman AW, Velasco R, Borodovsky M, Veilleux RE, Folta KM. The genome of woodland strawberry (Fragaria vesca). Nat Genet. 2011;43(2):109-16.

5. Star B, Nederbragt AJ, Jentoft S, Grimholt U, Malmstrøm M, Gregers TF, Rounge TB, Paulsen J, Solbakken MH, Sharma A, Wetten OF, Lanzén A, Winer R, Knight J, Vogel JH, Aken B, Andersen $\varnothing$, Lagesen K, Tooming-Klunderud A, Edvardsen RB, Tina KG, Espelund M, Nepal C, Previti C, Karlsen BO, Moum T, Skage M, Berg PR, Gjøen T, Kuhl H, Thorsen J, Malde K, Reinhardt R, Du L, Johansen SD, Searle S, Lien S, Nilsen F, Jonassen I, Omholt SW, Stenseth NC, Jakobsen KS. The genome sequence of Atlantic cod reveals a unique immune system. Nature. 2011;477(7363):207-10.

6. IWGSC TIWGSC, Mayer KFX, Rogers J, Doležel J, Pozniak C, Eversole K, Feuillet C, Gill B, Friebe B, Lukaszewski AJ, Sourdille P, Endo TR, Kubaláková M, Číhalíková J, Dubská Z, Vrána J, Šperková R, Šimková H, Febrer M, Clissold L, McLay K, Singh K, Chhuneja P, Singh NK, Khurana J, AkhunovE, Choulet F, Alberti A, Barbe V, Wincker P, Kanamori $H$, Kobayashi F, Itoh T, Matsumoto T, Sakai H, Tanaka T, Wu J, Ogihara Y, Handa H, Maclachlan PR, Sharpe A, Klassen D, Edwards D, Batley J, Olsen OA, Sandve SR, Lien S, Steuernagel B, Wulff B, Caccamo M, Ayling S, Ramirez-Gonzalez RH, Clavijo BJ, Wright J, Pfeifer M, Spannagl M, Martis MM, Mascher M, Chapman J, Poland JA, Scholz U, Barry K, Waugh R, Rokhsar DS, Muehlbauer GJ, Stein N, Gundlach H, Zytnicki M, Jamilloux V, Quesneville H, Wicker T, Faccioli P, Colaiacovo M, Stanca AM, Budak H, Cattivelli L, Glover N, Pingault L, PauxE, Sharma S, Appels R, Bellgard M, Chapman B, Nussbaumer T, Bader KC, Rimbert H, Wang S, Knox R, Kilian A, Alaux M, Alfama F, Couderc L, Guilhot N, Viseux C, Loaec M, Keller B, Praud S. A chromosome-based draft sequence of the hexaploid bread wheat (Triticum aestivum) genome. Science. 2014;345(6194):1251788.

7. Nystedt B, Street NR, Wetterbom A, Zuccolo A, Lin YC, Scofield DG, Vezzi F, Delhomme N, Giacomello S, Alexeyenko A, Vicedomini R, Sahlin K, Sherwood E, Elfstrand M, Gramzow L, Holmberg K, Hällman J, Keech O, Klasson L, Koriabine M, Kucukoglu M, Käller M, Luthman J, Lysholm F, Niittylä T, Olson Å, Rilakovic N, Ritland C, Rosselló JA, Sena J, Svensson T, Talavera-López C, Theißen G, Tuominen H, Vanneste K, Wu ZQ, Zhang B, Zerbe P, Arvestad L, Bhalerao R, Bohlmann J, Bousquet J, Garcia Gil R, Hvidsten TR, de Jong P, MacKay J, Morgante M, Ritland K, Sundberg B, Lee Thompson S, Van de Peer Y, Andersson B, Nilsson O, Ingvarsson PK, Lundeberg J, Jansson S. The Norway spruce genome sequence and conifer genome evolution. Nature. 2013;497(7451):579-84. 
8. Zhang G, Fang $X$, Guo $X$, Li L, Luo R, Xu F, Yang P, Zhang L, Wang $X$, Qi H, Xiong Z, Que H, Xie Y, Holland PWH, Paps J, Zhu Y, Wu F, Chen $Y$, Wang J, Peng C, Meng J, Yang L, Liu J, Wen B, Zhang N, Huang Z, Zhu Q, Feng Y, Mount A, Hedgecock D, Xu Z, Liu Y, Domazet-Lošo T, Du Y, Sun $X$, Zhang $S$, Liu B, Cheng $P$, Jiang $X$, Li J, Fan D, Wang W, Fu W, Wang T, Wang B, Zhang J, Peng Z, Li Y, Li N, Wang J, Chen M, He Y, Tan F, Song X, Zheng Q, Huang R, Yang H, Du X, Chen L, Yang M, Gaffney PM, Wang S, Luo L, She Z, Ming Y, Huang W, Zhang S, Huang B, Zhang Y, Qu T, Ni P, Miao G, Wang J, Wang Q, Steinberg CEW, Wang H, Li N, Qian L, Zhang G, Li Y, Yang H, Liu X, Wang J, Yin $Y$, Wang J. The oyster genome reveals stress adaptation and complexity of shell formation. Nature. 2012;490(7418):49-54.

9. Lien S, Koop BF, Sandve SR, Miller JR, Kent MP, Nome T, Hvidsten TR, Leong JS, Minkley DR, Zimin A, Grammes F, Grove H, Gjuvsland A, Walenz BP, Hermansen RA, von Schalburg K, Rondeau EB, Di Genova A, Samy JKA, Olav Vik J, Vigeland MD, Caler L, Grimholt U, Jentoft S, Inge Våge $\mathrm{D}$, de Jong $\mathrm{P}$, Moen $\mathrm{T}$, Baranski $\mathrm{M}$, Palti $\mathrm{Y}$, Smith DR, Yorke $J A$, Nederbragt AJ, Tooming-Klunderud A, Jakobsen KS, Jiang $X$, Fan D, Hu Y, Liberles DA, Vidal R, Iturra P, Jones SJM, Jonassen I, Maass A, Omholt SW, Davidson WS. The Atlantic salmon genome provides insights into rediploidization. Nature. 2016;533(7602):200-5.

10. Marcussen T, Sandve SR, Heier L, Spannagl M, Pfeifer M, International Wheat Genome Sequencing Consortium, Jakobsen KS, Wulff BBH, Steuernagel B, Mayer KFX, Olsen OA. Ancient hybridizations among the ancestral genomes of bread wheat. Science. 2014;345(6194):1250092.

11. Zhang G, Li C, Li Q, Li B, Larkin DM, Lee C, Storz JF, Antunes A, Greenwold MJ, Meredith RW, Ödeen A, Cui J, Zhou Q, Xu L, Pan H, Wang Z, Jin L, Zhang P, Hu H, Yang W, Hu J, Xiao J, Yang Z, Liu Y, Xie Q, Yu H, Lian J, Wen P, Zhang F, Li H, Zeng Y, Xiong Z, Liu S, Zhou L, Huang Z, An N, Wang J, Zheng Q, Xiong Y, Wang G, Wang B, Wang J, Fan $Y$, da Fonseca RR, Alfaro-Núñez A, Schubert M, Orlando L, Mourier T, Howard JT, Ganapathy G, Pfenning A, Whitney O, Rivas MV, Hara E, Smith J, Farré M, Narayan J, Slavov G, Romanov MN, Borges R, Machado JP, Khan I, Springer MS, Gatesy J, Hoffmann FG, Opazo JC, Håstad O, Sawyer RH, Kim H, Kim KW, Kim HJ, Cho S, Li N, Huang Y, Bruford MW, Zhan X, Dixon A, Bertelsen MF, Derryberry E, Warren W, Wilson RK, Li S, Ray DA, Green RE, O'Brien SJ, Griffin D, Johnson WE, Haussler D, Ryder OA, Willerslev E, Graves GR, Alström P, Fjeldsa J, Mindell DP, Edwards SV, Braun EL, Rahbek C, Burt DW, Houde P, Zhang Y, Yang H, Wang J, Consortium AG, Jarvis ED, Gilbert MTP, Wang J, Ye C, Liang S, Yan Z, Zepeda ML, Campos PF, Velazquez AMV, Samaniego JA, Avila-Arcos M, Martin MD, Barnett R, Ribeiro AM, Mello CV, Lovell PV, Almeida D, Maldonado E, Pereira J, Sunagar K, Philip S, Dominguez-Bello MG, Bunce M, Lambert D, Brumfield RT, Sheldon FH, Holmes EC, Gardner PP, Steeves TE, Stadler PF, Burge SW, Lyons E, Smith J, McCarthy F, Pitel F, Rhoads D, Froman DP. Comparative genomics reveals insights into avian genome evolution and adaptation. Science. 2014;346(6215):1311-20.

12. Jarvis ED, Mirarab S, Aberer AJ, Li B, Houde P, Li C, Ho SYW, Faircloth BC, Nabholz B, Howard JT, Suh A, Weber CC, da Fonseca RR, Li J, Zhang F, Li H, Zhou L, Narula N, Liu L, Ganapathy G, Boussau B, Bayzid MS, Zavidovych V, Subramanian S, Gabaldón T,

Capella-Gutiérrez S, Huerta-Cepas J, Rekepalli B, Munch K, Schierup M, Lindow B, Warren WC, Ray D, Green RE, Bruford MW, Zhan X, Dixon A, Li S, Li N, Huang Y, Derryberry EP, Bertelsen MF, Sheldon FH, Brumfield RT, Mello CV, Lovell PV, Wirthlin M, Schneider MPC, Prosdocimi F, Samaniego JA, Velazquez AMV, Alfaro-Núñez A, Campos PF, Petersen B, Sicheritz-Ponten T, Pas A, Bailey T, Scofield P, Bunce M, Lambert DM, Zhou Q, Perelman P, Driskell AC, Shapiro B, Xiong Z, Zeng Y, Liu S, Li Z, Liu B, Wu K, Xiao J, Yinqi X, Zheng Q, Zhang Y, Yang $\mathrm{H}$, Wang J, Smeds L, Rheindt FE, Braun M, Fjeldsa J, Orlando L, Barker FK, Jønsson KA, Johnson W, Koepfli KP, O’Brien S, Haussler D, Ryder OA, Rahbek C, Willerslev E, Graves GR, Glenn TC, McCormack J, Burt D, Ellegren H, Alström P, Edwards SV, Stamatakis A, Mindell DP, Cracraft J, Braun EL, Warnow T, Jun W, Gilbert MTP, Zhang G. Whole-genome analyses resolve early branches in the tree of life of modern birds. Science. 2014;346(6215):1320-31.

13. Alkan C, Sajjadian S, Eichler EE. Limitations of next-generation genome sequence assembly,. Nat Methods. 2011;8(1):61-5.

14. Chaisson MJP, Wilson RK, Eichler EE. Genetic variation and the de novo assembly of human genomes. Nature Rev Genet. 2015;16(11):627-40.
15. Treangen TJ, Salzberg SL. Repetitive DNA and next-generation sequencing: computational challenges and solutions,. Nature Rev Genet. 2012;13(1):36-46.

16. Miller JR, Koren S, Sutton GG. Assembly algorithms for next-generation sequencing data,. Genomics. 2010;95(6):315-27.

17. Chalopin D, Naville M, Plard F, Galiana D, Volff JN. Comparative analysis of transposable elements highlights mobilome diversity and evolution in vertebrates. Genome Biol Evol. 2015;7(2):567-80.

18. Mayer C, Leese F, Tollrian R. Genome-wide analysis of tandem repeats in Daphnia pulex - a comparative approach,. BMC Genom. 2010;11:277.

19. Gemayel R, Vinces MD, Legendre M, Verstrepen KJ. Variable tandem repeats accelerate evolution of coding and regulatory sequences. Annu Rev Genet. 2010;44(1):445-77.

20. Roberts RJ, Carneiro MO, Schatz MC. The advantages of SMRT sequencing. Genome Biol. 2013;14(6):405.

21. Koren S, Schatz MC, Walenz BP, Martin J, Howard JT, Ganapathy G, Wang Z, Rasko DA, McCombie WR, Jarvis ED, Phillippy AM. Hybrid error correction and de novo assembly of single-molecule sequencing reads. Nat Biotechnol. 2012;30(7):693-700.

22. Conte MA, Kocher TD. An improved genome reference for the African cichlid, Metriaclima zebra. BMC Genom. 2015;16(1):1.

23. Pendleton M, Sebra R, Pang AWC, Ummat A, Franzen O, Rausch T, Stütz AM, Stedman W, Anantharaman T, Hastie A, Dai H, Fritz MH-Y, Cao H, Cohain A, Deikus G, Durrett RE, Blanchard SC, Altman R, Chin CS, Guo Y, Paxinos EE, Korbel JO, Darnell RB, McCombie WR, Kwok PY, Mason CE, Schadt EE, Bashir A. Assembly and diploid architecture of an individual human genome via single-molecule technologies. Nat Methods. 2015;12(8):780-6.

24. Berlin K, Koren S, Chin CS, Drake JP, Landolin JM, Phillippy AM. Assembling large genomes with single-molecule sequencing and locality-sensitive hashing. Nat Biotechnol. 2015;33(6):623-30.

25. Vij S, Kuhl H, Kuznetsova IS, Komissarov A, Yurchenko AA, van Heusden P, Singh S, Thevasagayam NM, Prakki SRS, Purushothaman K, Saju JM, Jiang J, Mbandi SK, Jonas M, Tong AHY, Mwangi S, Lau D, Ngoh SY, Liew WC, Shen X, Hon LS, Drake JP, Boitano M, Hall R, Chin CS, Lachumanan R, Korlach J, Trifonov V, Kabilov M, Tupikin A, Green D, Moxon S, Garvin T, Sedlazeck FJ, Vurture GW, Gopalapillai G, Katneni VK, Noble TH, Scaria V, Sivasubbu S, Jerry DR, O'Brien SJ, Schatz MC, Dalmay T, Turner SW, Lok S, Christoffels A, Orban L. Chromosomal-level assembly of the Asian seabass genome using long sequence reads and multi-layered scaffolding. PLoS Genet. 2016;12(4):1005954.

26. Braasch I, Peterson SM, Desvignes T, McCluskey BM, Batzel P, Postlethwait JH. A new model army: Emerging fish models to study the genomics of vertebrate Evo-Devo. J Exp Zool B. 2015;324(4):316-41.

27. Flicek P, Amode MR, Barrell D, Beal K, Billis K, Brent S, Carvalho-Silva D, Clapham P, Coates G, Fitzgerald S, Gil L, Giron CG, Gordon L, Hourlier T, Hunt S, Johnson N, Juettemann T, Kahari AK, Keenan S, Kulesha E, Martin FJ, Maurel T, McLaren WM, Murphy DN, Nag R, Overduin B, Pignatelli M, Pritchard B, Pritchard E, Riat HS, Ruffier M, Sheppard D, Taylor K, Thormann A, Trevanion SJ, Vullo A, Wilder SP, Wilson M, Zadissa A, Aken BL, Birney E, Cunningham F, Harrow J, Herrero J, Hubbard TJP, Kinsella R, Muffato M, Parker A, Spudich G, Yates A, Zerbino DR, Searle SMJ. Ensembl 2014. Nucleic Acids Res. 2013;42(D1): 749-55.

28. Salzberg SL, Phillippy AM, Zimin A, Puiu D, Magoc T, Koren S, Treangen TJ, Schatz MC, Delcher AL, Roberts M, Marcais G, Pop M, Yorke JA. GAGE: A critical evaluation of genome assemblies and assembly algorithms. Genome Res. 2012;22(3):557-67.

29. Earl D, Bradnam K, St John J, Darling A, Lin D, Fass J, Yu HOK, Buffalo V, Zerbino DR, Diekhans M, Nguyen N, Ariyaratne PN, Sung WK, Ning Z, Haimel M, Simpson JT, Fonseca NA, Birol I, Docking TR, Ho IY, Rokhsar D, Chikhi R, Lavenier D, Chapuis G, Naquin D, Maillet N, Schatz MC, Kelley DR, Phillippy AM, Koren S, Yang SP, Wu W, Chou WC, Srivastava A, Shaw TI, Ruby JG, Skewes-Cox P, Betegon M, Dimon MT, Solovyev V, Seledtsov I, Kosarev P, Vorobyev D, Ramirez-Gonzalez R, Leggett R, MacLean D, Xia F, Luo R, Li Z, Xie Y, Liu B, Gnerre S, Maccallum I, Przybylski D, Ribeiro FJ, Yin S, Sharpe T, Hall G, Kersey PJ, Durbin R, Jackman SD, Chapman JA, Huang X, DeRisi JL, Caccamo M, Li Y, Jaffe DB, Green RE, Haussler D, Korf IF, Paten B. Assemblathon 1:a competitive assessment of de novo short read assembly methods. Genome Res. 2011;21(12):2224-41. 
30. Bradnam KR, Fass JN, Alexandrov A, Baranay P, Bechner M, Birol I, Boisvert S, Chapman JA, Chapuis G, Chikhi R, Chitsaz H, Chou WC, Corbeil J, Del Fabbro C, Docking T, Durbin R, Earl D, Emrich S, Fedotov P, Fonseca NA, Ganapathy G, Gibbs RA, Gnerre S, Godzaridis É, Goldstein S, Haimel M, Hall G, Haussler D, Hiatt JB, Ho IY, Howard J, Hunt M, Jackman SD, Jaffe DB, Jarvis ED, Jiang H, Kazakov S, Kersey PJ, Kitzman JO, Knight JR, Koren S, Lam TW, Lavenier D, Laviolette F, Li Y, Li Z, Liu B, Liu Y, Luo R, Maccallum I, MacManes MD, Maillet N, Melnikov $S$, Naquin D, Ning Z, Otto TD, Paten B, Paulo OS, Phillippy AM, Pina-Martins F, Place M, Przybylski D, Qin X, Qu C, Ribeiro FJ, Richards S, Rokhsar D, Ruby J, Scalabrin S, Schatz MC, Schwartz DC, Sergushichev A, Sharpe T, Shaw TI, Shendure J, Shi Y, Simpson JT, Song H, Tsarev F, Vezzi F, Vicedomini R, Vieira BM, Wang J, Worley KC, Yin S, Yiu SM, Yuan J, Zhang G, Zhang H, Zhou S, Korf IF.

Assemblathon 2: evaluating de novo methods of genome assembly in three vertebrate species. GigaScience. 2013;2(1):10.

31. Gnerre S, Maccallum I, Przybylski D, Ribeiro FJ, Burton JN, Walker BJ, Sharpe T, Hall G, Shea TP, Sykes S, Berlin AM, Aird D, Costello M, Daza R, Williams L, Nicol R, Gnirke A, Nusbaum C, Lander ES, Jaffe DB. High-quality draft assemblies of mammalian genomes from massively parallel sequence data. Proc Natl Acad Sci U S A. 2011;108(4):1513-8.

32. Miller JR, Delcher AL, Koren S, Venter E, Walenz BP, Brownley A, Johnson J, Li K, Mobarry C, Sutton GG. Aggressive assembly of pyrosequencing reads with mates. Bioinformatics. 2008;24(24):2818-24.

33. Walker BJ, Abeel T, Shea T, Priest M, Abouelliel A, Sakthikumar S, Cuomo CA, Zeng Q, Wortman J, Young SK, Earl AM. Pilon: An integrated tool for comprehensive microbial variant detection and genome assembly Improvement. PLOS ONE. 2014;9(11):112963.

34. English AC, Richards S, Han Y, Wang M, Vee V, Qu J, Qin X, Muzny DM, Reid JG, Worley KC, Gibbs RA. Mind the gap: upgrading genomes with Pacific Biosciences RS long-read sequencing technology. PLOS ONE. 2012;7(11):47768.

35. Vezzi F, Narzisi G, Mishra B. Reevaluating assembly evaluations with feature response curves: GAGE and Assemblathons. PLOS ONE. 2012;7(12):52210.

36. Hunt M, Kikuchi T, Sanders M, Newbold C, Berriman M, Otto TD. REAPR: a universal tool for genome assembly evaluation. Genome Biol. 2013;14(5):47.

37. Ryan JF. Baa.pl: a tool to evaluate de novo genome assemblies with RNA transcripts. ArXiv e-prints. 2013;1309.2087:1-9.

38. Parra G, Bradnam K, Korf IF. CEGMA: a pipeline to accurately annotate core genes in eukaryotic genomes. Bioinformatics. 2007;23(9):1061-7.

39. Simão FA, Waterhouse RM, loannidis P, Kriventseva EV, Zdobnov EM. BUSCO: assessing genome assembly and annotation completeness with single-copy orthologs. Bioinformatics. 2015;31(19):3210-2.

40. Yao G, Ye L, Gao H, Minx P, Warren WC, Weinstock GM. Graph accordance of next-generation sequence assemblies. Bioinformatics. 2011;28(1):13-16.

41. Wences $\mathrm{AH}$, Schatz MC. Metassembler: merging and optimizing de novo genome assemblies. Genome Biol. 2015;16(1):1.

42. Angiuoli SV, Salzberg SL. Mugsy: fast multiple alignment of closely related whole genomes. Bioinformatics. 2011;27(3):334-42.

43. Simpson JT, Durbin R. Efficient construction of an assembly string graph using the FM-index. Bioinformatics. 2010;26(12):367-73.

44. Simpson JT. Exploring genome characteristics and sequence quality without a reference. Bioinformatics. 2014;30(9):023-1235.

45. Hardie DC, Hebert P. The nucleotypic effects of cellular DNA content in cartilaginous and ray-finned fishes. Genome. 2003;46(4):683-706.

46. Hardie DC, Hebert PD. Genome-size evolution in fishes. Can J Fish Aquat Sci. 2004;61(9):1636-46.

47. Schartl M, Walter RB, Shen Y, Garcia T, Catchen J, Amores A, Braasch I, Chalopin D, Volff JN, Lesch KP, Bisazza A, Minx P, Hillier L, Wilson RK, Fuerstenberg S, Boore J, Searle S, Postlethwait JH, Warren WC. The genome of the platyfish, Xiphophorus maculatus, provides insights into evolutionary adaptation and several complex traits. Nat Genet. 2013:45(5):567-72

48. Rondeau EB, Minkley DR, Leong JS, Messmer AM, Jantzen JR, von Schalburg KR, Lemon C, Bird NH, Koop BF. The genome and linkage map of the northern pike (Esox lucius): conserved synteny revealed between the salmonid sister group and the Neoteleostei,. PLOS ONE. 2014;9(7):102089.
49. Campbell MS, Law M, Holt C, Stein JC, Moghe GD, Hufnagel DE, Lei J, Achawanantakun R, Jiao D, Lawrence CJ, Ware D, Shiu SH, Childs KL, Sun Y, Jiang N, Yandell M. MAKER-P: a tool kit for the rapid creation, management, and quality control of plant genome annotations. Plant Physiol. 2014;164(2):513-24.

50. Holt C, Yandell M. MAKER2: an annotation pipeline and genome-database management tool for second-generation genome projects. BMC Bioinform. 2011;12(1):491.

51. Tørresen OK, Samy JKA, Våge DI, Nederbragt AJ. A genome browser for the Atlantic cod genome version 2. http://www.mn.uio.no/cees/ english/genome-browser. Accessed 1 Mar 2016.

52. Li H. Aligning sequence reads, clone sequences and assembly contigs with BWA-MEM. ArXiv e-prints. 2013;1303.3997:1-3.

53. Garrison E, Marth G. Haplotype-based variant detection from short-read sequencing. ArXiv e-prints. 2012;1207.3907:1-9.

54. Chaisson MJP, Tesler G. Mapping single molecule sequencing reads using basic local alignment with successive refinement (BLASR): application and theory. BMC Bioinform. 2012;13(1):238.

55. English AC, Salerno WJ, Reid JG. PBHoney: Identifying genomic variants via long-read discordance and interrupted mapping. BMC Bioinform. 2014;15(1):180.

56. Smit A, Hubley R. RepeatModeler Open-1.0. http://www.repeatmasker. org. Accessed 30 Nov 2015.

57. Ellinghaus D, Kurtz S, Willhoeft U. LTRharvest, an efficient and flexible software for de novo detection of LTR retrotransposons. BMC Bioinform. 2008;9(1):1.

58. Steinbiss S, Willhoeft U, Gremme G, Kurtz S. Fine-grained annotation and classification of de novo predicted LTR retrotransposons. Nucleic Acids Res. 2009;37(21):7002-013.

59. Haas BJ. TransposonPSI. http://transposonpsi.sourceforge.net. Accessed 1 Feb 2016.

60. Jurka J, Kapitonov W, Pavlicek A, Klonowski P, Kohany O, Walichiewicz J. Repbase Update, a database of eukaryotic repetitive elements. Cytogenet Genome Res. 2005;110(1-4):462-7.

61. Jiang $Q$, Li Q, Yu H, Kong L. Genome-wide analysis of simple sequence repeats in marine animals - a comparative approach. Mar Biotechnol. 2014;16(5):604-19.

62. Gymrek M, Golan D, Rosset S, Erlich Y. lobSTR: A short tandem repeat profiler for personal genomes. Genome Res. 2012;22(6):1154-62.

63. Benson G. Tandem repeats finder: a program to analyze DNA sequences Nucleic Acids Res. 1999;27(2):573-80.

64. Willems T, Gymrek M, Highnam G, 1000 Genomes Project Consortium, Mittelman D, Erlich Y. The landscape of human STR variation. Genome Res. 2014;24(11):1894-904.

65. Parra G, Bradnam K, Ning Z, Keane T, Korf IF. Assessing the gene space in draft genomes. Nucleic Acids Res. 2009;37(1):289-97.

66. Vinson JP, Jaffe DB, O'Neill K, Karlsson EK, Stange-Thomann N, Anderson S, Mesirov JP, Satoh N, Satou Y, Nusbaum C, Birren B, Galagan JE, Lander ES. Assembly of polymorphic genomes: algorithms and application to Ciona savignyi. Genome Res. 2005;15(8):1127-35.

67. XuT, Xu G, Che R, Wang R, Wang Y, Li J, Wang S, Shu C, Sun Y, Liu T, Liu J, Wang A, Han J, Chu Q, Yang Q. The genome of the miiuy croaker reveals well-developed innate immune and sensory systems. Sci Rep. 2016;6:21902.

68. Jones FC, Grabherr MG, Chan YF, Russell P, Mauceli E, Johnson J, Swofford R, Pirun M, Zody MC, White S, Birney E, Searle S, Schmutz J, Grimwood J, Dickson MC, Myers RM, Miller CT, Summers BR, Knecht AK, Brady SD, Zhang H, Pollen AA, Howes T, Amemiya C, Baldwin J, Bloom T, Jaffe DB, Nicol R, Wilkinson J, Lander ES, Di Palma F, Lindblad-Toh K, Kingsley DM. The genomic basis of adaptive evolution in threespine sticklebacks. Nature. 2012;484(7392):55-61.

69. Martinez Barrio A, Lamichhaney S, Fan G, Rafati N, Pettersson M, Zhang H, Dainat J, Ekman D, Höppner M, Jern P, Martin M, Nystedt B, Liu X, Chen W, Liang X, Shi C, Fu Y, Ma K, Zhan X, Feng C, Gustafson U, Rubin CJ, Sällman Almén M, Blass M, Casini M, Folkvord A, Laikre L, Ryman N, Ming-Yuen Lee S, Xu X, Andersson L. The genetic basis for ecological adaptation of the Atlantic herring revealed by genome sequencing. eLife. 2016;5:12081.

70. Li H. Toward better understanding of artifacts in variant calling from high-coverage samples. Bioinformatics. 2014;30(20):2843-51. 
71. Jiang $Y$, Turinsky $A L$, Brudno M. The missing indels: an estimate of indel variation in a human genome and analysis of factors that impede detection. Nucleic Acids Res. 2015;43(15):677-7228.

72. Star B, Hansen MH, Skage M, Bradbury IR, Godiksen JA, Kjesbu OS, Jentoft S. Preferential amplification of repetitive DNA during whole genome sequencing library creation from historic samples. Sci Technol Archaeol Res. 2016;2(1):36-45.

73. Ellegren $\mathrm{H}$. Microsatellite mutations in the germline: implications for evolutionary inference. Trends Genet. 2000;16(12):551-8.

74. Ellegren H. Microsatellites: simple sequences with complex evolution. Nature Rev Genet. 2004;5(6):435-45.

75. Firtina $C$, Alkan C. On genomic repeats and reproducibility. Bioinformatics. 2016;32(15):2243-47.

76. Usdin K. The biological effects of simple tandem repeats: lessons from the repeat expansion diseases. Genome Res. 2008;18(7):1011-9.

77. Hammock EAD, Young LJ. Microsatellite instability generates diversity in brain and sociobehavioral traits. Science. 2005;308(5728):1630-4.

78. Fondon III JW, Garner HR. Molecular origins of rapid and continuous morphological evolution. Proc Natl Acad Sci U S A. 2004;101(52): 18058-63.

79. Sawaya S, Bagshaw A, Buschiazzo E, Kumar P, Chowdhury S, Black MA, Gemmell N. Microsatellite tandem repeats are abundant in human promoters and are associated with regulatory elements,. PLOS ONE. 2013;8(2):54710.

80. Vinces MD, Legendre M, Caldara M, Hagihara M, Verstrepen KJ. Unstable tandem repeats in promoters confer transcriptional evolvability. Science. 2009;324(5931):1213-6.

81. Gymrek M, Willems T, Guilmatre A, Zeng H, Markus B, Georgiev S, Daly MJ, Price AL, Pritchard JK, Sharp AJ, Erlich Y. Abundant contribution of short tandem repeats to gene expression variation in humans. Nat Genet. 2016;48(1):22-29.

82. Sonay TB, Carvalho T, Robinson M, Greminger M, Krutzen M, Comas D, Highnam G, Mittelman DA, Sharp AJ, Marques-Bonet T, Wagner A. Tandem repeat variation in human and great ape populations and its impact on gene expression divergence. Genome Res. 2015;25(11): 190868-51599.

83. Ohadi M, Valipour E, Ghadimi Haddadan S, Namdar Aligoodarzi P, Bagheri A, Kowsari A, Rezazadeh M, Darvish H, Kazeminasab S. Core promoter short tandem repeats as evolutionary switch codes for primate speciation. Am J Primatol. 2015;77(1):34-43.

84. Verstrepen KJ, Jansen A, Lewitter F, Fink GR. Intragenic tandem repeats generate functional variability. Nat Genet. 2005;37(9):986-90.

85. Lindqvist C, Laakkonen L, Albert VA. Polyglutamine variation in a flowering time protein correlates with island age in a Hawaiian plant radiation. BMC Evol Biol. 2007;7(1):1

86. Ottersen G, Bogstad B, Yaragina NA, Stige LC, Vikebo FB, Dalpadado P. A review of early life history dynamics of Barents Sea cod (Gadus morhua). ICES J Mar Sci. 2014;71(8):2064-87.

87. Poulsen NAA, Nielsen EE, Schierup MH, Loeschcke V, Grønkjaer P. Long-term stability and effective population size in North Sea and Baltic Sea cod (Gadus morhua). Mol Ecol. 2006;15(2):321-31.

88. Kent WJ. BLAT-the BLAST-like alignment tool. Genome Res. 2002;12(4): 656-64.

89. Magoc T, Salzberg SL. FLASH: fast length adjustment of short reads to improve genome assemblies. Bioinformatics. 2011;27(21):2957-63.

90. Prüfer K, Munch K, Hellmann I, Akagi K, Miller JR, Walenz BP, Koren S, Sutton GG, Kodira C, Winer R, Knight JR, Mullikin JC, Meader SJ, Ponting CP, Lunter G, Higashino S, Hobolth A, Dutheil J, Karakoç E, Alkan C, Sajjadian S, Catacchio CR, Ventura M, Marques-Bonet T, Eichler EE, André C, Atencia R, Mugisha L, Junhold J, Patterson N, Siebauer M, Good JM, Fischer A, Ptak SE, Lachmann M, Symer DE, Mailund T, Schierup MH, Andrés AM, Kelso J, Pääbo S. The bonobo genome compared with the chimpanzee and human genomes. Nature. 2012;486(7404):527-31.

91. Li H. Toolkit for processing sequences in FASTA/Q formats. https:// github.com/lh3/seqtk. Accessed 13 Sep 2015.

92. Li H, Handsaker B, Wysoker A, Fennell T, Ruan J, Homer N, Marth G, Abecasis G, Durbin R, 1000 Genome Project Data Processing Subgroup. The sequence alignment/map format and SAMtools. Bioinformatics. 2009;25(16):2078-9.
93. Kurtz S, Phillippy A, Delcher AL, Smoot M, Shumway M, Antonescu C, Salzberg SL. Versatile and open software for comparing large genomes. Genome Biol. 2004;5(2):12

94. Hubert S, Higgins B, Borza T, Bowman S. Development of a SNP resource and a genetic linkage map for Atlantic cod (Gadus morhua). BMC Genom. 2010;11(1):191.

95. Grabherr MG, Haas BJ, Yassour M, Levin JZ, Thompson DA, Amit I, Adiconis X, Fan L, Raychowdhury R, Zeng Q, Chen Z, Mauceli E, Hacohen N, Gnirke A, Rhind N, Di Palma F, Birren BW, Nusbaum C, Lindblad-Toh K, Friedman N, Regev A. Full-length transcriptome assembly from RNA-Seq data without a reference genome. Nat Biotechnol. 2011;29(7):644-52.

96. Margulies M, Egholm M, Altman WE, Attiya S, Bader JS, Bemben LA, Berka J, Braverman MS, Chen YJ, Chen Z, Dewell SB, Du L, Fierro JM, Gomes XV, Godwin BC, He W, Helgesen S, Ho CH, Irzyk GP, Jando SC, Alenquer MLI, Jarvie TP, Jirage KB, Kim JB, Knight JR, Lanza JR, Leamon JH, Lefkowitz SM, Lei M, Li J, Lohman KL, Lu H, Makhijani VB, McDade KE, McKenna MP, Myers EW, Nickerson E, Nobile JR, Plant R, Puc BP, Ronan MT, Roth GT, Sarkis GJ, Simons JF, Simpson JW, Srinivasan M, Tartaro KR, Tomasz A, Vogt KA, Volkmer GA, Wang SH, Wang Y, Weiner MP, Yu P, Begley RF, Rothberg JM. Genome sequencing in microfabricated high-density picolitre reactors. Nature. 2005;437(7057):376-80.

97. Gordon SP, Tseng E, Salamov A, Zhang J, Meng X, Zhao Z, Kang D, Underwood J, Grigoriev IV, Figueroa M, Schilling JS, Chen F, Wang Z. Widespread polycistronic transcripts in fungi revealed by single-molecule mRNA sequencing. PLOS ONE. 2015;10(7):0132628.

98. Penglase S, Edvardsen RB, Furmanek T, Rønnestad I, Karlsen $\varnothing$, van der Meeren T, Hamre K. Diet affects the redox system in developing Atlantic cod (Gadus morhua) larvae. Redox Biol. 2015;5:308-18.

99. Martin M. Cutadapt removes adapter sequences from high-throughput sequencing reads. EMBnet.journal. 2011;17(1):10-12.

100. Haas BJ, Papanicolaou A, Yassour M, Grabherr M, Blood PD, Bowden J, Couger MB, Eccles D, Li B, Lieber M, MacManes MD, Ott M, Orvis J, Pochet N, Strozzi F, Weeks N, Westerman R, William T, Dewey CN, Henschel R, LeDuc RD, Friedman N, Regev A. De novo transcript sequence reconstruction from RNA-seq using the Trinity platform for reference generation and analysis. Nat Protoc. 2013;8(8):1494-512.

101. Kleppe L, Edvardsen RB, Kuhl H, Malde K, Furmanek T, Drivenes $\varnothing$, Reinhardt R, Taranger GL, Wargelius A. Maternal 3'UTRs: from egg to onset of zygotic transcription in Atlantic cod. BMC Genom. 2012;13(1): 443.

102. Llorens C, Muñoz-Pomer A, Futami R. The GyDB collection of viral and mobile genetic element models. Biotechvana Bioinf. 2009;36:D38-D46.

103. Jiang N, Bowman M, Childs K. Repeat library construction-advanced. http://weatherby.genetics.utah.edu/MAKER/wiki/index.php/ Repeat_Library_Construction-Advanced. Accessed 21 Dec 2015.

104. Edgar RC. MUSCLE: multiple sequence alignment with high accuracy and high throughput,. Nucleic Acids Res. 2004;32(5):1792-7.

105. Kennedy RC, Unger MF, Christley S, Collins FH, Madey GR. An automated homology-based approach for identifying transposable elements. BMC Bioinform. 2011;12(1):1.

106. Fu L, Niu B, Zhu Z, Wu S, Li W. CD-HIT: accelerated for clustering the next-generation sequencing data. Bioinformatics. 2012;28(23):3150-2.

107. UniProt Consortium. UniProt: a hub for protein information. Nucleic Acids Res. 2015;43(Database issue):204-12.

108. Cantarel BL, Korf IF, Robb SMC, Parra G, Ross E, Moore B, Holt C, Sánchez Alvarado A, Yandell M. MAKER: an easy-to-use annotation pipeline designed for emerging model organism genomes,. Genome Res. 2008;18(1):188-96.

109. Kumar S. How to predict genes using a two-pass (iterative) MAKER2 workflow. https://github.com/sujaikumar/assemblage/blob/master/ README-annotation.md. Accessed 7 Aug 2015.

110. Campbell MS, Holt C, Moore B, Yandell M. Genome annotation and curation using MAKER and MAKER-P. Curr Protoc Bioinformatics. 2014;48:4-11141139.

111. Korf IF. Gene finding in novel genomes. BMC Bioinform. 2004;5(1):59.

112. Lomsadze A, Ter-Hovhannisyan V, Chernoff YO, Borodovsky M. Gene identification in novel eukaryotic genomes by self-training algorithm. Nucleic Acids Res. 2005;33(20):6494-506. 
113. Stanke M, Waack S. Gene prediction with a hidden Markov model and a new intron submodel. Bioinformatics. 2003;19(suppl 2):215-25.

114. Stanke M, Diekhans M, Baertsch R, Haussler D. Using native and syntenically mapped cDNA alignments to improve de novo gene finding. Bioinformatics. 2008;24(5):637-44.

115. Jones P, Binns D, Chang HY, Fraser M, Li W, McAnulla C, McWilliam H, Maslen J, Mitchell A, Nuka G, Pesseat S, Quinn AF, Sangrador-Vegas A, Scheremetjew M, Yong SY, Lopez R, Hunter S. InterProScan 5: genome-scale protein function classification. Bioinformatics. 2014;30(9):1236-40.

116. Yandell M, Ence D. A beginner's guide to eukaryotic genome annotation. Nature Rev Genet. 2012;13(5):329-42.

117. Quinlan AR, Hall IM. BEDTools: a flexible suite of utilities for comparing genomic features,. Bioinformatics. 2010;26(6):841-2

Submit your next manuscript to BioMed Central and we will help you at every step:

- We accept pre-submission inquiries

- Our selector tool helps you to find the most relevant journal

- We provide round the clock customer support

- Convenient online submission

- Thorough peer review

- Inclusion in PubMed and all major indexing services

- Maximum visibility for your research

Submit your manuscript at www.biomedcentral.com/submit
C) Biomed Central 